\title{
Differences in A $\beta$ brain networks in Alzheimer's disease and healthy controls
}

Huoqiang Duan ${ }^{1}$, Jiehui Jiang ${ }^{1}$, Jun $\mathrm{Xu}^{1}$, Hucheng Zhou ${ }^{1}$, Zhemin Huang ${ }^{2}$, Zhihua $\mathrm{Yu}^{3 *}$, Zhuangzhi Yan ${ }^{1}$ and The Alzheimer's Disease Neuroimaging Initiative.

\begin{abstract}
The prevailing $\beta$-amyloid (A $\beta$ )-cascade hypothesis is the most classical Alzheimer's disease (AD) pathogenesis. In this hypothesis, excessive $A \beta$ plaque deposition in human brain is considered to be the cause of AD. Carbon 11-labeled Pittsburgh compound B Positron emission tomography (11C-PiB PET) is the latest technology to detect $\mathrm{A} \beta$ plaques in vivo. Thus, it is possible to investigate the difference of $\mathrm{A} \beta$ brain networks between AD patients and Health Controls (HC) by analyzing 11C-PiB PET images. In this study, a graph-theoretical method was employed to investigate the topological properties of $\mathrm{A} \beta$ networks in 18 Chinese $\mathrm{AD}$ patients and $16 \mathrm{HC}$ subjects from Huashan Hospital, Shanghai. The results showed that both groups demonstrated small-world property, and this property was more obvious in AD group. Additionally, the clustering coefficients and path lengths were significantly lower in AD group. The global efficiency was larger in AD than in HC. A direct comparison between with and without regression found that sex, age and weight had no significant effect on the $A \beta$ network. Moreover, three altered regions in AD group were identified, including left cuneus (CUN.L), right caudate nucleus (CAU.R) and left superior frontal gyrus (SFGdor.L). A voxel-wise correlation analysis showed that in AD patients, the regions of strengthened connection with CUN.L were mainly located in frontal cortex and parietal cortex, the regions of strengthen connection with CAU.R were mainly located in temporal cortex. Finally, a machine learning based analysis demonstrated that the three regions could be better biomarkers than the whole brain for $\mathrm{AD}$ classification.
\end{abstract}

Keywords: Alzheimer's disease, $\beta$-amyloid (A $\beta)$-cascade, 11C-PiB PET, brain networks, graph theoretical 
Alzheimer's disease (AD) is the most common form of dementia. People who suffer from $\mathrm{AD}$ frequently experience memory loss and other intellectual disabilities, which interferes with their daily lives (Piccininni et al., 2005).

Although AD pathogenesis remains unclear, a few hypotheses have been widely recognized. The most classical hypothesis is the prevailing $\beta$-amyloid (A $\beta$ )-cascade hypothesis that was proposed in 1991 by John Hardy and David Allsop (Hardy and Allsop, 1991). They suggested that the mismetabolism of the amyloid precursor protein (APP) was the initiating event in AD pathogenesis, subsequently leading to the aggregation and deposition of $A \beta$, specifically $A \beta 42$ in the brain parenchyma (Nobuyuki et al., 2008). Consequently, this process causes a series of variations in brain tissues, including brain oxidative stress, neural vascular barrier dysfunction, depressed metabolism, synaptic membranes lesions, and neural cell death, that ultimately leads to Alzheimer's disease (Zlokovic, 2005). Based on this foundation, several assumptions have been proposed over the past ten years (Domert et al., 2014; Guo and Lee, 2014; Thal, 2015). One assumption that has been proposed is that $\mathrm{A} \beta$ deposits are mutually influential rather than act independently between brains regions, even when the deposits are located far away from one another. The interactions between connected neurons are responsible for this phenomenon at the brain structural level (Sepulcre et al., 2013).

Carbon 11-labeled Pittsburgh compound B Positron emission tomography (11C-PiB PET) is one of the latest clinical technologies to detect $A \beta$ plaques deposition in human brains in vivo. Over the past decade, several research groups have utilized 11C-PiB PET imaging to study AD pathogenesis and to explore diagnostic significances between AD patients and healthy control subjects. For instance, research groups have explored the variations of $\mathrm{A} \beta$ plaques in different brain areas for both $\mathrm{AD}$ patients and $\mathrm{HC}$ subjects as well as have identified imaging biomarkers of $\mathrm{PiB}$ PET images (Grimmer et al., 2009; Ikonomovic et al., 2008; Price et al., 2005; Rabinovici et al., 2007). Various traditional methods, such as the T test, Logan analysis, and more, have been used for this purpose (Mikhno et al., 2008; Tolboom et al., 2009).

Although some initial findings have been obtained, these findings need to be further validated and studied. As a complex integrative system in which billions of neurons connect with one another in an extremely complicated way, human brains continuously process and transport information between spatially distributed, but functionally linked, regions (van den Heuvel et al., 2009). However, traditional statistical methods did not take the connections between different brain regions into consideration; thus, a new method is required to optimize the current findings. Brain networks analysis may be a good alternative for addressing such studies.

Brain networks analysis is based on the graph-theoretical method, and it provides a mathematic model for discovering the structural and functional connections of cells 
and fibers in human brains. As early as 1984, network-based methods have been applied to fluorodeoxyglucose (FDG) PET images for metabolic networks research (Horwitz B Fau - Duara et al., 1984). With the continuous optimization of the method itself as well as the development of various imaging techniques, brain networks analysis has gradually become a hot research topic in neuroscience, and a series of results in mental and neurological brain diseases and cognitive research has been achieved using this method (Leistedt et al., 2009; Liu et al., 2016; Rudie et al., 2013; Sanz-Arigita et al., 2010; Schindler et al., 2008). As the most common form of dementia, AD study is a popular implementation of brain networks analysis. Several research groups have applied the brain network method to investigate brain alterations and cognitive deficits associated with the AD process (He et al., 2008; Lo et al., 2010; Supekar et al., 2008; Wang et al., 2013). For example, neuroscientists found that tau protein could spread through neural circuits in human brains (de Calignon et al., 2012; Liu et al., 2012), thus connectivity mapping studies with PET-tau has become blossoming in the $\mathrm{AD}$ community right now. Literature showed that tau protein pathology may develop in vulnerable networks, such as the posterior Default Mode Network (DMN), and then spread into closely interconnected networks (Clavaguera et al., 2009; Frost et al., 2009).

Similar to PET-tau studies, in recent years, a few studies have begun to apply PiB PET technology and the brain network method to construct $A \beta$ brain networks for AD research. For instance, Sepulcre et al. proposed that $A \beta$ aggregation in the medial temporal lobe is associated with aggregation in cortical regions, such as the orbitofrontal cortex and lateral temporal lobe (Sepulcre et al., 2013). Jiang et al. analyzed datasets from the Alzheimer's Disease Neuroimaging Initiative (ADNI) database and proposed that the left medial superior frontal cortex, right medial orbital wall, and frontal inferior parietal lobe could be potential A $\beta$ seeds in AD (Jiang et al., 2015). Song et al. applied the network analysis method and proposed that a cortical $\mathrm{A} \beta$ load in cognitively normal elderly is associated with disrupted functional connectivity of the perirhinal cortex (Song et al., 2015). Son et al. performed a connectivity analysis combining FDG PET and PiB PET images for mild cognitive impairment (MCI) subjects and proposed five regions wherein significant changes in betweenness centrality were observed. (Son et al., 2015). Leyton et al. analyzed A $\beta$ brain networks properties in amnestic $\mathrm{AD}$ and a logopenic-variant of primary progressive aphasia (lv-PPA) cases with a high cortical A $\beta$ burden. As a result, it was proposed that regional neurotoxic effects may explain the phenotypical differences in $\mathrm{AD}$ presentation and can be modulated by the apolipoprotein E (APOE) genotype (Leyton et al., 2016).

Nevertheless, even though brain networks analysis has been applied to construct A $\beta$ brain networks in previous studies, improvements are still required to make up for aspects that were disregarded. First, previous studies used Pearson correlation method without considering the impact of sex, age and weight (Jiang et al., 2015; Sepulcre et al., 2013). Second, brain $A \beta$ network properties in AD had not been compared with HCs comprehensively. Third, the biomarkers of $A \beta$ in human brains are still required 
to be identified.

This study therefore concentrates on four main objectives: (1) to investigate whether sex, age and weight had an effect on the $A \beta$ network, (2) to explore the $A \beta$ network connectivity patterns in $A D$ patients and the physiological significance of $A \beta$ brain networks comprehensively, (3) to identify altered hubs of $A \beta$ brain networks and locate the significant correlation regions with altered hubs in $\mathrm{AD}$, in order to explore how $A \beta$ spreads throughout the whole human brain, and (4) to investigate whether these altered hubs can serve as valuable biomarkers of $\mathrm{A} \beta$ for $\mathrm{AD}$ classification. Finally, the outcomes of this paper are expected to fulfill the $A \beta$ cascade hypothesis.

\section{Results}

\subsection{Impact of sex, age and weight on the A $\beta$ network}

A direct comparison between networks with and without regressing out the factors of sex, age and weight had been done in this study. Figure 2 shows the results of $\mathrm{C}, \mathrm{L}$ and Sigma. Both in the AD group and HC group, the results showed that the difference between with and without regressing is very little. As a result, no matter regressing or without regressing the factors of sex, age and weight, clustering coefficient and path length in $\mathrm{AD}$ group were lower than in $\mathrm{HC}$ group, whereas sigma in $\mathrm{AD}$ group was larger than in $\mathrm{HC}$ group.

\subsection{Network Parameters}

Figure 3 shows the results of gamma, lambda, and sigma in the AD group and HC group by using partial correlation matrices. As shown in Figure 3(C), over the entire threshold range of $6 \%-40 \%$, both $\mathrm{AD}$ and $\mathrm{HC}$ groups fulfilled gamma $>>1$, lambda $\approx 1$, and sigma $>1$, which means both networks demonstrated small-world property in this study. However, this property was more obvious and noticeable in the AD group than in the HC group. For example, at $18 \%$ sparsity, the value of sigma in the $\mathrm{AD}$ group reached 1.64, while it was 1.15 only in the HC group. Further permutation testing showed that over the entire threshold range, the gamma and sigma in the AD group were significantly larger than in the HC group, whereas lambda was lower in the AD group than in the HC group $(\mathrm{p}<0.05)$.

As shown in Figure 4, the network parameters of C, L, globalE, and localE in the two groups likewise changed quite a bit. In Figure 4 (A), parameter $\mathrm{C}$ gradually becomes larger as sparsity increases; the $\mathrm{HC}$ group increases from 0.32 to 0.59 , and the $\mathrm{AD}$ group increases from 0.32 to 0.54 over the entire threshold range. The statistical analysis by permutation test revealed that parameter $\mathrm{C}$ was significantly lower in the AD group than in the HC group at certain sparsity values; those being $10 \%-14 \%$ and $21 \%(\mathrm{p}<0.05)$. In Figure 4 (B), the parameter L value in the HC group decreases from 5.03 to 1.43 , and in the $\mathrm{AD}$ group, it decreases from 3.60 to 1.43 as the sparsity increases over the entire range. Similar to the above network results for parameter $\mathrm{C}$, the statistical analysis revealed that parameter L was significantly lower in the AD 
group than in the HC group at certain sparsity values; those being $8 \%-10 \%$ and $12 \%$ - 14\% (p < 0.05). Figure 4 (C), (D) shows the results for globalE and localE. The globalE value was significantly larger in the $\mathrm{AD}$ group than in the $\mathrm{HC}$ group at sparsity values of $8 \%-10 \%, 13 \%$, and $14 \%(p<0.05)$. Finally, the localE value in the $\mathrm{AD}$ group was approximately equal to that of the $\mathrm{HC}$ group, and there was no significant change over the entire threshold range.

\subsection{Hubs regions}

In order to determine the hubs in the $\mathrm{HC}$ and $\mathrm{AD}$ groups, a fixed sparsity of $18 \%$ was chosen to calculate the network parameter of normalized betweenness centrality. $18 \%$ was selected because $18 \%$ is the lowest density where the largest component size was 90 in the present study. It should be noted that, by calculating the component size of the two networks separately, the minimum sparsity thresholds that ensured that the network without isolated nodes for the $\mathrm{HC}$ and $\mathrm{AD}$ groups were $18 \%$ and $13 \%$, respectively. Figure 5 shows the largest component size of the two networks as a function of the sparsity threshold. Finally, the specific sparsity of $18 \%$ was chosen, and the bi values of each node in the two networks were calculated. Such a constraint might optimize the interregional correlation strengths and might be biologically plausible (He et al., 2008; Seo et al., 2013a).

At sparsity of 18\%, 21 hub nodes were identified in the HC group, and 18 hub nodes were identified in the AD group. Table 3 and Table 4 list the bi values and functional classification of the hub regions in the $\mathrm{HC}$ group and $\mathrm{AD}$ group, respectively. Functionally, the hubs in both groups are primarily located in association areas. Figure 6 shows the hub nodes results in the axial view. Anatomically, the hubs in HC are primarily located in the frontal and temporal cortex, and the hubs in AD are also located in the occipital cortex besides the frontal and temporal. In both groups, five regions were identified as hubs in common, including the right middle frontal gyrus (MFG.R), left middle orbitofrontal (ORBmid.L), left supramarginal gyrus (SMG.L), left caudate putamina (CAU.L), and superficial temporal (STG.L).

Furthermore, in the between-group comparisons, statistical analysis with permutation test showed that three brain regions significantly changed in the AD group $(p<0.05)$, including left superior frontal gyrus (SFGdor.L), left cuneus (CUN.L) and right caudate nucleus (CAU.R). Figure 7 shows these three regions with the medium view and axial view. Figure 8 shows the bi values and the $\mathrm{p}$ values of the three regions. In Figure 8, it can be observed that SFGdor.L was the hub node in the HC group, and CUN.L and CAU.R were hub nodes in the AD group. More information on all hub nodes can be found in Appendix Table 3.

\subsection{Seed ROI-based correlation}

To further investigate the detailed connectivity associated with the hubs in the two groups, the brain regions CUN.L and CAU.R were selected as seeds in this study. These regions were selected because of two primary reasons. First, both CUN.L and 
CAU.R were identified as altered hubs in AD (i.e., the value of normalized bi was greater than 1.5). Second, the statistical analysis by permutation test shows that for both CUN.L and CAU.R, the bi values were significantly different between the AD and $\mathrm{HC}$ groups (for CUN.L, $\mathrm{p}$ value $=0.006$; for CAU.R, $\mathrm{p}$ value $=0.044$ ). Details of the bi and $\mathrm{p}$ values for 90 nodes are described in Appendix Table 3.

Figure 9 demonstrates the results of the voxel-wise correlation analysis with CUN.L seed. Figure 9 (A), (B) shows the correlation coefficients map (R-map) associated with the CUN.L seed in HC and AD, respectively. In the HC group, the results of the R-map showed that the areas that present positive connections with the CUN.L seed are mainly located in the left precentral gyrus (PreCG.L), right superior frontal gyrus (SFGdor.R), and right supplementary motor area (SMA.R). However, in the AD group, most brain regions were positively correlated with CUN.L. Further analysis by $\mathrm{Z}$ statistics in the R-map showed that the regions of strengthened connection with CUN.L in the AD group that were located in the frontal cortex included the left precentral gyrus (PreCG.R), left midcingulate area (DCG.L), SFGdor.R and SMA.R, and in the parietal cortex included the left postcentral gyrus (PoCG.L), right postcentral gyrus (PoCG.R) and left inferior parietal lobule (IPL.L) ( $p<0.05$, FDR corrected). Figure $9(\mathrm{C})$ shows the $\mathrm{Z}$ statistics results; the highlighted areas represent brain regions with strengthened connection with CUN.L in the AD group.

Figure 10 shows the results of the voxel-wise correlation analysis with the CAU.R seed in the two groups. The R-map comparisons showed that in the AD group, the regions of enhanced connection with CAU.R were primarily located in left parahippocampal gyrus (PHG.L), right parahippocampal gyrus (PHG.R), right middle temporal pole (TPOmid.R), and right inferior temporal gyrus (ITG.R) $(\mathrm{p}<0.05$, FDR corrected).

\subsection{Altered hubs validation}

To validate whether SFGdor.L, CUN.L, and CAU.R can serve as biomarkers for AD diagnosis, a separate dataset including $30 \mathrm{HC}$ and $30 \mathrm{AD}$ patients selected from ADNI database was used for altered hubs validation. Specifically, PCA method was used to obtain the image features of the three altered hubs, and SVM was used for AD classification. In this study, sigmoid kernel was used for SVM, and the classification performance was achieved with an Acc of $85.0 \%$, Sen of $90.0 \%$, and Spc of $80.0 \%$, respectively.

As contrast experiments, a single brain region of SFGdor.L, CUN.L, and CAU.R and 90 regions of the whole brain were also studied for AD classification. As shown in Table 5, the classification performance for the whole brain achieved an Acc of 83.3\%, Sen of $90.0 \%$, and Spc of $76.7 \%$, respectively. This performance is better than the results of using a single brain region and is worse than the performance of using the three altered hubs for AD classification. Figure 11 shows the ROC curves of five sets of biomarkers. The AUC values of SFGdor.L, CUN.L, CAU.R, the three hubs, and the whole brain were $0.52,0.63,0.68,0.86$, and 0.78 , respectively. The results again 
indicated that the three altered hubs had the best performance and could be better biomarkers for $\mathrm{AD}$ diagnosis and recognition.

\section{Discussion}

Using 11C-PiB PET images and graph-based network analysis methods, the characteristics of the whole-brain $\mathrm{A} \beta$ brain networks were investigated in the $\mathrm{HC}$ and AD groups. The main findings are as follows: (1) sex, age and weight had no significant effect on the A $\beta$ network; (2) both the HC and AD groups had small-world property, but this property was more significant in the AD group; (3) the clustering coefficient and characteristic path length was larger in the HC group; (4) the global efficiency was larger in the AD group, but the local efficiency was similar; (5) three altered hubs were found: SFGdor.L, CUN.L, and CAU.R; and (6) a machine learning based analysis demonstrated that the three altered hubs could be better biomarkers for $\mathrm{AD}$ classification. In this section, the physiological and pathological meanings of these findings are discussed.

\subsection{Impact of sex, age and weight on the $A \beta$ network}

According to previous studies, aging is not only a major risk factor but a major determinant of onset, course, and pathogenesis for AD (Bondareff, 2013). Direct evidence also showed that the $A \beta$ plaque burden significantly increased with age (Hanna et al., 2012; Patterson et al., 2015). However, in the present study, a direct comparison between networks with and without regressing out the factors of sex, age and weight had been done. Different from previous findings, we found sex, age and weight had no significant effect on the $A \beta$ network. As can be found in Table 1, the observed p-values of sex, age and weight between AD group and HC group were $0.15,0.41$ and 0.06 , respectively. These results indicated that there is no obvious difference between $\mathrm{AD}$ group and $\mathrm{HC}$ group in sex, age and weight. Consequently, regressing the factors or not will not cause notable influence on the $\mathrm{A} \beta$ network. Nevertheless, these results were limited by using present datasets. Deeper investigations on the effect of sex, age and weight for the $A \beta$ network need to be done in future.

\subsection{Network parameters of $\mathrm{HC}$ and AD}

In this study, although both the $\mathrm{HC}$ and $\mathrm{AD}$ groups demonstrated small-world network property, the results of the network parameters revealed that the AD group experienced great changes as compared with the HC group. Specifically, the AD group demonstrated shorter path lengths and lower clustering coefficients across the overwhelming majority thresholds. In addition, more noticeable small-world property was also observed in the $\mathrm{AD}$ group. Taking these results into consideration, we may propose an explanation from the respect of $A \beta$-cascade hypothesis.

As mentioned in the introduction, evidence from previous studies have shown that $A \beta$ proteins were able to quickly spread between different neuronal cells, and the 
capability of $\mathrm{A} \beta$ generation and expansion will be increased for $\mathrm{AD}$ patients as compared to $\mathrm{HC}$ subjects. As a result, leading to more brain regions exist $\mathrm{A} \beta$ plaques in the brain of $\mathrm{AD}$ patient. Owing to PiB PET images reflect the distribution of $\mathrm{A} \beta$ plaques in the human brain, thus $A \beta$ network connection enhanced and lead to a noticeable small-world property.

For clustering coefficient, it is based on correlations between adjacent brain regions, and they reflect the degree of node aggregation (Seo et al., 2013b). Because the ability of $\mathrm{A} \beta$ plaques to spread is significantly stronger in $\mathrm{AD}$ patients, $\mathrm{A} \beta$ plaques can easily spread to remote brain regions. As a result, this phenomenon is reflected at the network level. The AD group exhibited a lower clustering coefficient and a weaker local specialization, which suggests that $A \beta$ plaques do not aggregate together and easily transport to different brain regions.

In terms of path length, it measures the ability of information transmission across brain regions. A low path length indicates there is shorter information transmission path between two nodes (Sporns and Zwi, 2004). In AD patients, A $\beta$ plaques are able to spread to other remote brain regions. As a result, although two regions far away from each other, their correlation might be strong. At the network level, a strong correlation means exists connection between the regions. Therefore, shorter paths can be achieved from one node to another remote node, and eventually causing AD group exhibited a lower path length. This suggests that shorter $A \beta$ transmission paths can be observed between two brain regions in AD patients.

It is necessary to note that the findings in this study are somewhat conflictive with a previous study (Jiang et al., 2015), in which it was proposed that AD patients had higher clustering coefficients, higher path lengths, and lower small-worldness. The conflicting results may be explained from $\mathrm{PiB}+$ and $\mathrm{PiB}-$. In this study, all the healthy controls were $\mathrm{PiB}$ - and all the $\mathrm{AD}$ patients were $\mathrm{PiB}+$, but they were not controlled in the previous study. The previous study used the ADNI dataset, in which more than 41 percent of HCs are classified as $\mathrm{PiB}+$ rather than PiB- (Perrotin et al., 2012). These $\mathrm{PiB}+\mathrm{HCs}$ may have some impact on the $\mathrm{A} \beta$ network analysis.

\subsection{Hubs of $A \beta$ brain networks}

In this study, 21 hub nodes were identified in the HC group, and 18 were identified in the AD group. These findings are similar to the results of previous studies on $A \beta$ brain networks. For instance, bilateral orbitofrontal cortex and right middle temporal cortex had been identified as hub regions of $\mathrm{HC}$ and AD group respectively (Leyton et al., 2016; Sepulcre et al., 2013). However, compared with hub regions from other metabolic and structure brain networks, the hub regions identified in this study may be different. This phenomenon is reasonable because $A \beta$ brain networks focuses on $\mathrm{A} \beta$ deposition. Direct evidence from previous study had showed that regions altered in the hierarchy between atrophy, hypometabolism, and A $\beta$ deposition were different (La Joie et al., 2012). 
In addition, three brain regions that significantly changed in the $\mathrm{AD}$ group were found. Among them, SFGdor.L showed a lower bi value while CUN.L and CAU.R showed larger bi values. These three hubs could be important biomarkers in AD diagnosis and play crucial roles in AD pathogenesis. CAU.R has already been proven to be an important biomarker in 11C-PiB PET images by calculating SUV (Price et al., 2005). This indicates that the results of the altered regions found by the graph-theoretical analysis method have similarities with the quantitative analyses in clinics. A study for High Angular Resolution Diffusion Imaging (HARDI) also showed that the centrality of CAU.R was more increased in the AD group than in the HC group (Wang et al., 2016). This means CAU.R regions exists in an abnormal topological organization in both the brain structure and $A \beta$ generation function. However, SFGdor.L and CUN.L are new findings that have not yet been reported in other studies.

Finally, the AD classification results in this study demonstrated that the three altered hubs can serve as valuable biomarkers for $\mathrm{AD}$ diagnosis and aid physicians in understanding $\mathrm{AD}$ pathogenesis in the future.

\subsection{Seed ROI-based correlation analysis}

The altered brain regions CUN.L and CAU.R were selected as seeds in this study. Seed ROI-based correlation analysis was performed to investigate the connectivity details of other brain regions that were associated with the altered hubs. The results showed that in the $\mathrm{AD}$ group, the regions of strengthened connection with CUN.L were mainly located in frontal cortex including PreCG.R, SFGdor.R, SMA.R, DCG.L and parietal cortex including PoCG.L, PoCG.R, IPL.L regions, while the regions of strengthen connection with CAU.R seed were mainly located in temporal cortex included the PHG.L, PHG.R, TPOmid.R, and ITG.R regions. As far as the A $\beta$ spread mechanism is concerned, we may assume that in AD patients, $A \beta$ accumulation in the CUN.L region is associated with accumulation in frontal cortex and parietal cortex, while $A \beta$ accumulation in the CAU.R region is associated with accumulation in temporal cortex. These findings could be useful for neuroscientists to further understand $A \beta$ spread patterns.

\subsection{Limitations and Future Questions}

There are several issues that still need to be further considered in this study. First, although many studies have applied the AAL template to explore functional connection patterns in the human brain, whether the scale of AAL template is suitable for $A \beta$ network analysis is still debatable. In future, we plan to use higher-resolution parcellation templates (Bai et al., 2012; Sun et al., 2014) to construct A $\beta$ network. Second, an unweighted and binary network was constructed in this study, which cannot reveal the direction of $A \beta$ spread. In future, we plan to construct directed networks for better understanding the mechanism of A $\beta$ spread. Third, partial correlation matrices were used to calculate the network parameters and identify the altered ROIs, subsequently, Pearson correlation was applied in this study for 
ROI-based correlation analysis. This may bring bias for the results. In future, we plan to further investigate the differences by using partial correlation and Pearson correlation matrices, respectively.

\section{Conclusion}

Based on brain network analysis for PiB PET images, this study systematically explored the whole-brain $A \beta$ network and $A \beta$ spread patterns in $\mathrm{HC}$ and $\mathrm{AD}$ groups. As a whole, both the AD and HC groups demonstrated small-world topology, but the topological organization of the $\mathrm{A} \beta$ network in $\mathrm{AD}$ group was significantly changed as compared with HC group. The abnormal topological connection is primarily reflected in the decreased clustering coefficients and path length as well as in the increased global efficiency. In addition, a direct comparison between networks found that sex, age and weight had no significant effect on the $A \beta$ network. Finally, this study identified three altered regions and demonstrated connectivity details of other brain regions associated with the altered hubs, which may assist clinicians in the AD diagnosis and provide help for neuroscientists to further understand $A \beta$ spread patterns.

\section{Experimental Procedure}

\subsection{Subjects}

Two datasets of 11C-PiB PET images were used as materials in this study. One dataset is from Shanghai Huashan Hospital PET Center and another one is from ADNI database. The first dataset was used for $\mathrm{A} \beta$ brain networks analysis, and the second one was used for altered hubs validation.

\subsubsection{Dataset from Shanghai Huashan Hospital}

Thirty-four right-handed subjects from the Shanghai Huashan Hospital PET Center were included in this study, including 18 AD patients and 16 HCs. Standardized uptake value ratios (SUVR) of PiB were calculated by dividing the cerebellar cortex standardized uptake values. Using 1.6 as the cutoff value of SUVR, 18 AD patients were confirmed as $\mathrm{PiB}+(\mathrm{PiB}$ positive $)$ objects, and $16 \mathrm{HCs}$ were confirmed as $\mathrm{PiB}-$ (PiB negative) objects. The statistical information of the 34 participants is summarized in Table 1, and each subject's clinical data is described in Appendix Table 1.

\subsubsection{Dataset from ADNI database}

Sixty subjects from the ADNI database were used for altered hubs validation, including $30 \mathrm{AD}$ patients and $30 \mathrm{HCs}$, which were selected from 300+ datasets in total by two senior clinicians from Shanghai Huashan hospital. For these 60 subjects, all PiB PET images from the $\mathrm{AD}$ patients were verified as $\mathrm{PiB}+$, and the images from the HCs were verified as PiB-. The statistical information of the 60 subjects is also summarized in Table 1, and each subject's clinical data is described in Appendix 
Table 2.

\subsection{PET Image Acquisition and Preprocessing}

The whole brain PET images for thirty-four right-handed subjects were acquired using the Siemens Biograph 64 in the PET Center, Huashan Hospital, Shanghai, China. The spatial resolution of the PET scanner is $5.9 \mathrm{~mm}$ full-width at half-maximum (FWHM) in the transaxial plane and $5.5 \mathrm{~mm}$ FWHM in the axial plane. All subjects were injected intravenously with $370 \mathrm{MBq}(10 \mathrm{mCi})$ [11C] $\mathrm{PiB}$ in a dimly-lit, quiet room with their eyes closed for 40-50 minutes so as to minimize the confounding effects of any activity. After that, static emission scans were continued for 20 minutes. The transaxial images were reconstructed as $168 \times 168 \times 148$ matrices with a size of $2.0 \times 2.0 \times 1.5 \mathrm{~mm}$ by using a filtered back-projection algorithm employing a Shepp-Logan filter. The acquisitions were performed with the approval of the institutional review board of Huashan Hospital and all participants signed informed consents.

All raw images were in the Digital Imaging and Communications in Medicine (DICOM) format. DCM2NII (https://www.nitrc.org/projects/dcm2nii/) was used to convert images from the DICOM to the NIfTI format. The transformed images were pre-processed by using Statistical Parametric Mapping 8 (SPM8) implemented in Matlab2014a. Firstly, all of the PET images were spatially normalized to the Montreal Neurological Institute (MNI, McGill University, Montreal, Canada) space by using the 'Normalise: Estimate and Write'. In this step, the individual images were spatially warped to a reference PET template from SPM software. This spatially warping is a completely automated procedure that using affine transformation with 12 parameters. Then, the normalized images were smoothed by convolution using an isotropic Gaussian kernel with $8 \times 8 \times 8 \mathrm{~mm}^{3} \mathrm{FWHM}$. Finally, the images were transferred to gray level images with a grayscale of $[0,255]$.

\subsection{Constructing the brain network}

In the graph theory, a network consists of vertices and edges that connect a sequence of vertices (Bullmore and Sporns, 2009). In our study, the graph theory-based approaches were used to construct the $\mathrm{A} \beta$ brain networks of the AD and $\mathrm{HC}$ groups.

The Standardized Automated Anatomical Labeling (AAL) template (90 brain regions in total, 45 in each cerebral hemisphere) was used to extract the brain regions (Tzourio-Mazoyer et al., 2002; Wang et al., 2009). Using the AAL template, all of the PiB PET images were divided into 90 nodes (one region was considered as one node). Subsequently, the average voxel intensity value of each node was calculated, which was considered as the node value in the brain network. Then, the value of each node was globally normalized to a zero-mean and unit-variance by subtracting the mean and dividing by the standard deviation of each individual's whole brain. According to prior studies (Lo et al., 2010; Stam et al., 2007), the interregional correlation matrix $(90 \times 90)$ was acquired by calculating the partial correlation coefficients between every 
pair of nodes across individuals. In this study, a partial correlation analysis was conducted for the control of the age, gender, and weight effects. Figure 1 (A), (B) shows the partial correlation matrix of the $\mathrm{HC}$ group and $\mathrm{AD}$ group, respectively.

In order to determine whether connections exist between two nodes, the sparsity threshold method was applied in this study (Bullmore and Sporns, 2009; Liu et al., 2014; Sun et al., 2014). Because there is no definitive way to select a single threshold, a wide range of sparsity threshold was applied, and the network parameters were calculated at each threshold. The threshold selection rule is according to the following equation (He et al., 2007): $K \geq \ln (\mathrm{N})$, where $K$ refers to the mean degree of the resulting network, $\mathrm{N}$ refers to the number of network nodes, and $\mathrm{N}$ is 90 . Finally, the sparsity threshold range of $6 \%-40 \%$ was chosen with an interval of $1 \%$. It should be noted that, how to deal with negative correlations when thresholding is still controversial. In this study, if the absolute value of negative correlation was greater than threshold, it would be converted to 1 when thresholding (Wang et al., 2015).

After thresholding, the correlation coefficient matrix was transformed into a binary matrix that was described as a network. In the binary matrix, an element of 1 indicates that there is a connection between two nodes, and 0 indicates that there is no connection, as shown in Figure 1 (C), (D).

\subsection{Network parameters analysis}

To explore differences in the network patterns between $\mathrm{AD}$ and $\mathrm{HC}$ groups, the following factors were analyzed in this study: the network parameters of clustering coefficient (C), characteristic path length (L), gamma, lambda, small-world coefficient (sigma), local efficiency (localE), global efficiency (globalE), and node betweenness centrality (BC). All parameters were calculated by the open toolkit GRETNA (https://www.nitrc.org/projects/gretna/) (Wang et al., 2015) and The Brain Connectivity Toolbox (BCT, http://www.nitrc.org/projects/bct/) (Rubinov and Sporns, 2010).

In the graph theory, the clustering coefficient, $\mathrm{Ci}$, of a node, $\mathrm{i}$, quantifies the number of connections that exist in the cluster as a proportion of the maximum possible connections (Watts and Strogatz, 1998). The C of a network refers to the average clustering coefficient of all nodes in the network, which measures the degree to which nodes in a graph tend to cluster together (Inoue and Liu, 2015). In the network, the shortest path is defined as the minimum number of edges to connect two nodes. The characteristic path length, L, of a network is the average of the shortest path of all pairs of nodes in the network, it is a measure of the efficiency of the information or mass transport of a network (He et al., 2007). It should be pointed out that, as path length of the disconnected node is infinity, in this study, harmonic mean path length (also called average inverse path length) was calculated as characteristic path length.

A small-world network should meet the following criteria: gamma $=\mathrm{C} / \mathrm{C}_{\text {rand }}>>1$, lambda $=\mathrm{L} / \mathrm{L}_{\text {rand }} \approx 1$ and sigma $=$ gamma $/$ lambda $>1$, where $\mathrm{C}_{\text {rand }}$ and $\mathrm{L}_{\text {rand }}$ refer 
to the clustering coefficient and the characteristic path length of a corresponding random network, respectively (Maslov and Sneppen, 2002; Watts and Strogatz, 1998). The random network was derived from the original brain network by randomly rewiring the edges between nodes while preserving the same number of nodes, edges, and degrees. In this study, random networks were repeated 200 times. In addition, the global efficiency (globeE) and local efficiency (localE) were also calculated. The efficiency of a network is a measure of how efficiently it exchanges information (Latora and Marchiori, 2001). For global efficiency, it is defined as the inverse of the harmonic mean of the shortest path length between each pair of nodes. For local efficiency, it reflects the information exchange efficiency between subgraphs (Achard and Bullmore, 2007).

Additionally, the Betweenness Centrality (BC) is an indicator of a node's centrality in a network. It is equal to the number of shortest paths from all vertices to all others that pass through that node. $\mathrm{BC}$ is normally used to determine the candidate hubs in a network (Bullmore and Sporns, 2009). In this study, the normalized parameter bi was defined by bi $=$ BC/averaged BC. According to (Seo et al., 2013b), nodes with high bi values (bi $>1.5$ ) were considered as candidate hubs of the $\mathrm{A} \beta$ brain networks. Then, in order to identify which hub region was significantly altered in the AD group as compared with the HC group, a nonparametric permutation test was applied with 2000 repetitions, and a statistical result with $\mathrm{p}<0.05$ was considered as altered hubs (see Materials and methods, Statistical analysis).

To facilitate easy access to all network parameters, the parameters are summarized in Table 2.

\subsection{Seed ROI-based correlation analysis}

After determining the altered hubs of the two groups, the seed ROI-based correlation analysis method was performed to further investigate the connectivity details of other brain regions that are associated with the identified altered hubs.

First of all, the Pearson correlation coefficient of each voxel in whole brain with the identified altered hubs was calculated. Then, the resulting correlation coefficients were transformed to the z-value using Fisher's r-to-z transformation, which ensures an approximate normal distribution. It was calculated as the following equation:

$$
z_{i}=1 / 2 \times \log \left[\left(1+r_{i}\right) /\left(1-r_{i}\right)\right]
$$

where $r_{i}$ refers to the correlation coefficients, and $\mathrm{zi}$ is the transformed $\mathrm{z}$-value. Finally, these $\mathrm{z}$-values were compared between groups by $\mathrm{Z}$ statistics using:

$$
z=\left(z_{1}-z_{2}\right) / \sqrt{1 /\left(n_{1}-3\right)+1 /\left(n_{2}-3\right)}
$$

where $\mathrm{n}_{1}$ and $\mathrm{n}_{2}$ refers to the samples of two groups (He et al., 2008; Zhu et al., 2012). To adjust for the multiple comparisons, a false discovery rate (FDR) procedure was 
performed at a q value of 0.05 (Genovese et al., 2002).

\subsection{Statistical analysis}

To determine the statistical significance of the differences in the network parameters between the AD group and $\mathrm{HC}$ group, a nonparametric permutation test with 2000 repetitions was used (He et al., 2008; Seo et al., 2013b).

First, to test the null hypothesis that the group differences might have occurred by accident, each participant's 90 node values were randomly reassigned to one of two groups, and the partial correlation coefficient matrix for each randomized group was recomputed. Then, the corresponding binarized matrix was obtained using the same sparsity threshold, and the same method was used to recalculate the network parameters of C, L, gamma, lambda, sigma, localE, globalE, and bi for each randomized group. This randomization procedure was repeated 2000 times at every sparsity threshold, and the 95 percentile scores of each difference distribution were considered as the critical values ( $p<0.05$, one-tailed).

\subsection{PCA and SVM for classification}

To investigate whether the altered hubs can serve as valuable biomarkers for AD classification, the Principal Component Analysis (PCA) method together with the Support Vector Machines (SVM) classifier were used in this study. PCA is a statistical procedure that uses an orthogonal transformation to convert original data into a set of linearly uncorrelated variables called principal components. It is mostly used as a tool in exploratory data analysis and for constructing predictive models. In general, PCA can be explained as the following equation (I.T.Jolliffe, 2002; Zuendorf et al., 2003):

$$
\mathrm{C}=\frac{1}{N} \sum_{1}^{N} y_{i} y_{i}^{T}=\frac{1}{N} Y Y^{T}
$$

where $\mathrm{C}$ is the covariance matrix of the normalized vectors set; $\mathrm{N}$ is the number of subjects; and $\mathrm{Y}=\left[\mathrm{Y}_{1}, \mathrm{Y}_{2}, \ldots, \mathrm{Y}_{\mathrm{n}}\right]$ is a vector set of subjects, where $\mathrm{Y}_{\mathrm{i}}=\left[\mathrm{y}_{\mathrm{i} 1}, \mathrm{y}_{\mathrm{i} 2}, \ldots, \mathrm{y}_{\mathrm{in}}\right]$ represents an $\mathrm{n}$-dimensional normalized vector, $\mathrm{i}=1,2, \ldots, \mathrm{n}$.

In machine learning, SVM is widely used for data classification and pattern recognition in many applications, it trains a model that can assign new input data into one category or the other. The general SVM model can be explained as follows (Cortes and Vapnik, 1995):

$$
\mathrm{D}=\left\{(x, y) \mid x_{i} \in R^{N}, y_{i} \in\{-1,1\}\right\}_{i=1}^{n}
$$

where $\mathrm{D}$ is training data that consists of $\mathrm{x}$ and $\mathrm{y}$, where $\mathrm{x}$ refers to the $\mathrm{N}$-dimensional input data of the subject, and $y$ is either 1 or -1 and refers to the corresponding label. 
In this study, the PCA function from Matlab 2013a was directly used. The PCA protocol can be divided into two steps. First, the gray level images with a grayscale [0-255] of 60 whole brain PET images from ADNI were achieved according to section 2.2, and the gray level images of SFGdor.L, CUN.L, CAU.R and joint images of three altered hubs were extracted using hubs' masks. Consequently, five groups of gray level images were identified, including whole brain group, SFGdor.L group, CUN.L group, CAU.R group, and three altered hubs group. Each group includes 60 subjects; Second, voxel values of 60 subjects in each group were inputted to PCA, respectively. As results, each subject in every group obtained 59 principal components (PCs), which represent the most relevant image information (color, texture, and shape) of PiB PET images.

Then, five SVM classifiers with linear kernels for five groups were employed using LIBSVM toolbox (Chang and Lin, 2011), respectively. For each group, the SVM protocol is composed of three steps. First, AD subjects were labelled as "1", and HC subjects were labelled as " -1 ". Second, in order to train SVM classifiers, the "leave one out" cross validation method was used with the consideration of the small samples in this study. In this step, 59 subjects were used as training data and 1 subject was used as testing data. For the training data, labels together with 59 PCs used as inputs of SVM classifiers, for the testing data, input its PCs and corresponding predicted label can be obtained. To guarantee every subject was tested, we carried out classification experiments in 60 times and obtained 60 testing results; Third, we compared 60 predicted labels and real labels, and finally achieved classification results. Accuracy (Acc), Sensitivity (Sen), and Specificity (Spc) were used to evaluate the results. The mathematic expressions of these three parameters are as follows:

$$
\begin{gathered}
\text { Accuracy }=\frac{T p+T n}{T p+T n+F p+F n} \\
\text { Sensitivity }=\frac{T p}{T p+F n} \\
\text { Specificity }=\frac{T n}{T n+F p}
\end{gathered}
$$

where Tp, Tn, Fp, and Fn denote true positives, true negatives, false positives, and false negatives, respectively.

In addition, in order to more intuitively compare the classification performances between the different biomarkers, the receiver operating characteristic (ROC) curves were calculated, and the area under the ROC curves (AUC) were computed by utilizing the decision values and predict labels from the SVM classifiers.

\section{Acknowledgements}

This research was supported by National Natural Science Foundation of China 
61603236, Scientific Research Foundation for Returned Scholars, Ministry of Education of China, and Scientific Research Foundation for excellent young scholars, Shanghai Municipal Education Commission. The authors would like to thank Mr. Xinghui Shu for his kindly help with data preprocessing.

Data collection and sharing for this project was funded by the Alzheimer's Disease Neuroimaging Initiative (ADNI) (National Institutes of Health Grant U01 AG024904) and DOD ADNI (Department of Defense award number W81XWH-12-2-0012). ADNI is funded by the National Institute on Aging, the National Institute of Biomedical Imaging and Bioengineering, and through generous contributions from the following: AbbVie, Alzheimer's Association; Alzheimer's Drug Discovery Foundation; Araclon Biotech; BioClinica, Inc.; Biogen; Bristol-Myers Squibb Company; CereSpir, Inc.; Eisai Inc.; Elan Pharmaceuticals, Inc.; Eli Lilly and Company; EuroImmun; F. Hoffmann-La Roche Ltd and its affiliated company Genentech, Inc.; Fujirebio; GE Healthcare; IXICO Ltd.; Janssen Alzheimer Immunotherapy Research \& Development, LLC.; Johnson \& Johnson Pharmaceutical Research \& Development LLC.; Lumosity; Lundbeck; Merck \& Co., Inc.; Meso Scale Diagnostics, LLC.; NeuroRx Research; Neurotrack Technologies; Novartis Pharmaceuticals Corporation; Pfizer Inc.; Piramal Imaging; Servier; Takeda Pharmaceutical Company; and Transition Therapeutics. The Canadian Institutes of Health Research is providing funds to support ADNI clinical sites in Canada. Private sector contributions are facilitated by the Foundation for the National Institutes of Health (www.fnih.org). The grantee organization is the Northern California Institute for Research and Education, and the study is coordinated by the Alzheimer's Disease Cooperative Study at the University of California, San Diego. ADNI data are disseminated by the Laboratory for Neuro Imaging at the University of Southern California.

\section{$7 \quad$ Reference}

Achard, S., Bullmore, E., 2007. Efficiency and Cost of Economical Brain Functional Networks. PLOS Computational Biology. 3, http://dx.doi.org/10.1371/journal.pcbi.0030017.

Bai, F., Shu N Fau - Yuan, Y., Yuan Y Fau - Shi, Y., Shi Y Fau - Yu, H., Yu H Fau - Wu, D., Wu D Fau - Wang, J., Wang J Fau - Xia, M., Xia M Fau - He, Y., He Y Fau - Zhang, Z., Zhang, Z., 2012. Topologically convergent and divergent structural connectivity patterns between patients with remitted geriatric depression and amnestic mild cognitive impairment. The Journal of Neuroscience. 32, 4307-4318.

Bondareff, W., 2013. Age-related changes in brain extracellular space affect processing of amyloid-beta peptides in Alzheimer's disease. Journal of Alzheimer's Disease. 35, 1-6.

Bullmore, E., Sporns, O., 2009. Complex brain networks: graph theoretical analysis of structural and functional systems. Nature Reviews Neuroscience. 10, 186-198.

Chang, C.-C., Lin, C.-J., 2011. LIBSVM: A library for support vector machines. ACM Trans. Intell. Syst. Technol. 2, 1-27.

Clavaguera, F., Bolmont, T., Crowther, R.A., Abramowski, D., Frank, S., Probst, A., Fraser, G., Stalder, A.K., Beibel, M., Staufenbiel, M., Jucker, M., Goedert, M., Tolnay, M., 2009. 
Transmission and spreading of tauopathy in transgenic mouse brain. Nat Cell Biol. 11, 909-913.

Cortes, C., Vapnik, V., 1995. Support-vector networks. Machine Learning. 20, 273-297.

de Calignon, A., Polydoro M Fau - Suarez-Calvet, M., Suarez-Calvet M Fau - William, C., William C Fau - Adamowicz, D.H., Adamowicz Dh Fau - Kopeikina, K.J., Kopeikina Kj Fau - Pitstick, R., Pitstick R Fau - Sahara, N., Sahara N Fau - Ashe, K.H., Ashe Kh Fau - Carlson, G.A., Carlson Ga Fau - Spires-Jones, T.L., Spires-Jones Tl Fau - Hyman, B.T., Hyman, B.T., 2012. Propagation of tau pathology in a model of early Alzheimer's disease. Neuron. 73, 685-697.

Domert, J., Rao, S.B., Agholme, L., Brorsson, A.-C., Marcusson, J., Hallbeck, M., Nath, S., 2014. Spreading of amyloid- $\beta$ peptides via neuritic cell-to-cell transfer is dependent on insufficient cellular clearance. Neurobiology of Disease. 65, 82-92.

Frost, B., Jacks Rl Fau - Diamond, M.I., Diamond, M.I., 2009. Propagation of tau misfolding from the outside to the inside of a cell. JOURNAL OF BIOLOGICAL CHEMISTRY. 289, 12845-12852.

Genovese, C.R., Lazar, N.A., Nichols, T., 2002. Thresholding of Statistical Maps in Functional Neuroimaging Using the False Discovery Rate. NeuroImage. 15, 870-878.

Grimmer, T., Henriksen, G., Wester, H.-J., Förstl, H., Klunk, W.E., Mathis, C.A., Kurz, A., Drzezga, A., 2009. Clinical severity of Alzheimer's disease is associated with PIB uptake in PET. Neurobiology of Aging. 30, 1902-1909.

Guo, J.L., Lee, V.M.Y., 2014. Cell-to-cell transmission of pathogenic proteins in neurodegenerative diseases. Nature Medicine. 20, 130-138.

Hanna, A., Iremonger, K., Das, P., Dickson, D., Golde, T., Janus, C., 2012. Age-related increase in amyloid plaque burden is associated with impairment in conditioned fear memory in CRND8 mouse model of amyloidosis. Alzheimer's Research \& Therapy. 4, 1-11.

Hardy, J., Allsop, D., 1991. Amyloid deposition as the central event in the aetiology of Alzheimer's disease. Trends in Pharmacological Sciences. 12, 383-388.

He, Y., Chen, Z.J., Evans, A.C., 2007. Small-World Anatomical Networks in the Human Brain Revealed by Cortical Thickness from MRI. Cereb Cortex. 17, 2407-2419.

He, Y., Chen, Z., Evans, A., 2008. Structural insights into aberrant topological patterns of large-scale cortical networks in Alzheimer's disease. The Journal of Neuroscience. 28, 4756-4766.

Horwitz B Fau - Duara, R., Duara R Fau - Rapoport, S.I., Rapoport, S.I., 1984. Intercorrelations of glucose metabolic rates between brain regions: application to healthy males in a state of reduced sensory input. Journal of Cerebral Blood Flow \& Metabolism. 4, 484-499.

I.T.Jolliffe, 2002. Principal Component Analysis. Vol., Springer, New York.

Ikonomovic, M.D., Klunk, W.E., Abrahamson, E.E., Mathis, C.A., Price, J.C., Tsopelas, N.D., Lopresti, B.J., Ziolko, S., Bi, W., Paljug, W.R., Debnath, M.L., Hope, C.E., Isanski, B.A., Hamilton, R.L., DeKosky, S.T., 2008. Post-mortem correlates of in vivo PiB-PET amyloid imaging in a typical case of Alzheimer's disease. Brain. 131, 1630-1645.

Inoue, H., Liu, Y.-Y., 2015. Revealing the Intricate Effect of Collaboration on Innovation. PLoS ONE. 10, http://dx.doi.org/10.1371/journal.pone.0121973.

Jiang, J., Duan, H., Huang, Z., Yu, Z., 2015. Study of amyloid-beta peptide functional brain networks in AD, MCI and HC. Bio-medical materials and engineering. 26, S2197-S2205.

La Joie, R., Perrotin A Fau - Barre, L., Barre L Fau - Hommet, C., Hommet C Fau - Mezenge, F., Mezenge F Fau - Ibazizene, M., Ibazizene M Fau - Camus, V., Camus V Fau - Abbas, A., 
Abbas A Fau - Landeau, B., Landeau B Fau - Guilloteau, D., Guilloteau D Fau - de La Sayette, V., de La Sayette V Fau - Eustache, F., Eustache F Fau - Desgranges, B., Desgranges B Fau - Chetelat, G., Chetelat, G., 2012. Region-specific hierarchy between atrophy, hypometabolism, and beta-amyloid (Abeta) load in Alzheimer's disease dementia. The Journal of Neuroscience. 32, 16265-16273.

Latora, V., Marchiori, M., 2001. Efficient Behavior of Small-World Networks. Physical Review Letters. 87, 1-4.

Leistedt, S.J., Coumans N Fau - Dumont, M., Dumont M Fau - Lanquart, J.-P., Lanquart Jp Fau - Stam, C.J., Stam Cj Fau - Linkowski, P., Linkowski, P., 2009. Altered sleep brain functional connectivity in acutely depressed patients. Human Brain Mapping. 30, 2207-2219.

Leyton, C.E., Cassidy, B., Villemagne, V.L., Jones, G., Kwok, J.B., Rowe, C.C., Ballard, K.J., Piguet, O., Hodges, J.R., 2016. Divergent Network Patterns of Amyloid-beta Deposition in Logopenic and Amnestic Alzheimer's Disease Presentations. Biological Psychiatry: Cognitive Neuroscience and Neuroimaging. 1, 24-31.

Liu, F., Zhuo, C., Yu, C., 2016. Altered cerebral blood flow covariance network in schizophrenia. Frontiers in Neuroscience. 10, http://dx.doi.org/10.3389/fnins.2016.00308.

Liu, L., Drouet, V., Wu, J.W., Witter, M.P., Small, S.A., Clelland, C., Duff, K., 2012. Trans-Synaptic Spread of Tau Pathology In Vivo. PLoS ONE. http://dx.doi.org/10.1371/journal.pone.0031302

Liu, Z., Ke, L., Liu, H., Huang, W., Hu, Z., 2014. Changes in topological organization of functional PET brain network with normal aging. PLoS One. 9, http://dx.doi.org/10.1371/ /journal.pone.0088690.

Lo, C.Y., Wang, P.N., Chou, K.H., Wang, J., He, Y., Lin, C.P., 2010. Diffusion tensor tractography reveals abnormal topological organization in structural cortical networks in Alzheimer's disease. The Journal of Neuroscience. 30, 16876-16885.

Maslov, S., Sneppen, K., 2002. Specificity and Stability in Topology of Protein Networks. Science. 296, 910-913.

Mikhno, A., Devanand, D., Pelton, G., Cuasay, K., Gunn, R., Upton, N., Lai, R.Y., Libri, V., Mann, J.J., Parsey, R.V., 2008. Voxel-Based Analysis of (11)C-PIB Scans for Diagnosing Alzheimer's Disease. Journal of nuclear medicine : official publication, Society of Nuclear Medicine. 49, 1262-1269.

Nobuyuki, O., Shozo, F., Hiroyuki, A., Ren, I., Kazuhiko, Y., Yukitsuka, K., 2008. Imaging Amyloid Pathology in the Living Brain. Current Medical Imaging Reviews. 4, 56-62.

Patterson, B.W., Elbert, D.L., Mawuenyega, K.G., Kasten, T., Ovod, V., Ma, S., Xiong, C., Chott, R., Yarasheski, K., Sigurdson, W., Zhang, L., Goate, A., Benzinger, T., Morris, J.C., Holtzman, D., Bateman, R.J., 2015. Age and amyloid effects on human central nervous system amyloid-beta kinetics. Annals of Neurology. 78, 439-453.

Perrotin, A., Mormino Ec Fau - Madison, C.M., Madison Cm Fau - Hayenga, A.O., Hayenga Ao Fau Jagust, W.J., Jagust, W.J., 2012. Subjective cognition and amyloid deposition imaging: a Pittsburgh Compound B positron emission tomography study in normal elderly individuals. Archives of Neurology. 69, 223-229.

Piccininni, M., Di Carlo, A., Baldereschi, M., Zaccara, G., Inzitari, D., 2005. Behavioral and Psychological Symptoms in Alzheimer's Disease: Frequency and Relationship with Duration and Severity of the Disease. Dementia and Geriatric Cognitive Disorders. 19, 276-281. 
Price, J.C., Klunk, W.E., Lopresti, B.J., Lu, X., Hoge, J.A., Ziolko, S.K., Holt, D.P., Meltzer, C.C., DeKosky, S.T., Mathis, C.A., 2005. Kinetic modeling of amyloid binding in humans using PET imaging and Pittsburgh Compound-B. J Cereb Blood Flow Metab. 25, 1528-1547.

Rabinovici, G.D., Furst Aj Fau - O'Neil, J.P., O'Neil Jp Fau - Racine, C.A., Racine Ca Fau - Mormino, E.C., Mormino Ec Fau - Baker, S.L., Baker Sl Fau - Chetty, S., Chetty S Fau - Patel, P., Patel P Fau - Pagliaro, T.A., Pagliaro Ta Fau - Klunk, W.E., Klunk We Fau - Mathis, C.A., Mathis Ca Fau - Rosen, H.J., Rosen Hj Fau - Miller, B.L., Miller Bl Fau - Jagust, W.J., Jagust, W.J., 2007. 11C-PIB PET imaging in Alzheimer disease and frontotemporal lobar degeneration. Neurology. 68, 1205-1212.

Rubinov, M., Sporns, O., 2010. Complex network measures of brain connectivity: uses and interpretations. Neuroimage. 52, 1059-1069.

Rudie, J.D., Brown, J.A., Beck-Pancer, D., Hernandez, L.M., Dennis, E.L., Thompson, P.M., Bookheimer, S.Y., Dapretto, M., 2013. Altered functional and structural brain network organization in autism. NeuroImage: Clinical. 2, 79-94.

Sanz-Arigita, E.J., Schoonheim, M.M., Damoiseaux, J.S., Rombouts, S.A.R.B., Maris, E., Barkhof, F., Scheltens, P., Stam, C.J., 2010. Loss of'Small-World'Networks in Alzheimer's Disease: Graph Analysis of fMRI Resting-State Functional Connectivity. PLoS ONE. 5, http://dx.doi.org/ 10.1371/journal.pone.0013788.

Schindler, K.A., Bialonski S Fau - Horstmann, M.-T., Horstmann Mt Fau - Elger, C.E., Elger Ce Fau Lehnertz, K., Lehnertz, K., 2008. Evolving functional network properties and synchronizability during human epileptic seizures. Chaos. 18,http://dx.doi.org/10.1063/ 1.2966112 .

Seo, E.H., Lee, D.Y., Lee, J.M., Park, J.S., Sohn, B.K., Choe, Y.M., Byun, M.S., Choi, H.J., Woo, J.I., 2013a. Influence of APOE genotype on whole-brain functional networks in cognitively normal elderly. PLoS One. 8, http://dx.doi.org/10.1371/ journal. pone.0083205.

Seo, E.H., Lee, D.Y., Lee, J.M., Park, J.S., Sohn, B.K., Lee, D.S., Choe, Y.M., Woo, J.I., 2013 b. Whole-brain functional networks in cognitively normal, mild cognitive impairment, and Alzheimer's disease. PLoS One. 8, http://dx.doi.org/10.1371/journal.pone.0053922.

Sepulcre, J., Sabuncu, M.R., Becker, A., Sperling, R., Johnson, K.A., 2013. In vivo characterization of the early states of the amyloid-beta network. Brain. 136, 2239-2252.

Son, S.J., Kim, J., Seo, J., Lee, J.M., Park, H., Adni, 2015. Connectivity analysis of normal and mild cognitive impairment patients based on FDG and PiB-PET images. Neuroscience Research. 98, 50-58.

Song, X.-W., Dong, Z.-Y., Long, X.-Y., Li, S.-F., Zuo, X.-N., Zhu, C.-Z., He, Y., Yan, C.-G., Zang, Y.-F., 2011. REST: A Toolkit for Resting-State Functional Magnetic Resonance Imaging Data Processing. PLoS ONE. 6, http://dx.doi.org/10.1371/journal.pone.0025031.

Song, Z., Insel, P.S., Buckley, S., Yohannes, S., Mezher, A., Simonson, A., Wilkins, S., Tosun, D., Mueller, S., Kramer, J.H., Miller, B.L., Weiner, M.W., 2015. Brain amyloid-beta burden is associated with disruption of intrinsic functional connectivity within the medial temporal lobe in cognitively normal elderly. J Neurosci. 35, 3240-3247.

Sporns, O., Zwi, J.D., 2004. The small world of the cerebral cortex. Neuroinformatics. 2, 145-162.

Stam, C.J., Jones, B.F., Nolte, G., Breakspear, M., Scheltens, P., 2007. Small-world networks and functional connectivity in Alzheimer's disease. Cereb Cortex. 17, 92-99. 
Sun, Y., Yin, Q., Fang, R., Yan, X., Wang, Y., Bezerianos, A., Tang, H., Miao, F., Sun, J., 2014. Disrupted functional brain connectivity and its association to structural connectivity in amnestic mild cognitive impairment and Alzheimer's disease. PLoS One. 9, http://dx.doi.org/10.1371/journal.pone.0096505.

Supekar, K., Menon, V., Rubin, D., Musen, M., Greicius, M.D., 2008. Network Analysis of Intrinsic Functional Brain Connectivity in Alzheimer's Disease. PLoS Computational Biology. 4, http://dx.doi.org/10.1371/journal.pcbi.1000100.

Thal, D.R., 2015. Clearance of amyloid $\beta$-protein and its role in the spreading of Alzheimer's disease pathology. Frontiers in Aging Neuroscience. 7, 1-4.

Tolboom, N., Yaqub, M., Boellaard, R., Luurtsema, G., Windhorst, A.D., Scheltens, P., Lammertsma, A.A., Berckel, B.N.M., 2009. Test-retest variability of quantitative [11C]PIB studies in Alzheimer's disease. European Journal of Nuclear Medicine and Molecular Imaging. 36, $1629-1638$.

Tzourio-Mazoyer, N., Landeau, B., Papathanassiou, D., Crivello, F., Etard, O., Delcroix, N., Mazoyer, B., Joliot, M., 2002. Automated anatomical labeling of activations in SPM using a macroscopic anatomical parcellation of the MNI MRI single-subject brain. Neuroimage. 15, 273-289.

van den Heuvel, M.P., Stam Cj Fau - Kahn, R.S., Kahn Rs Fau - Hulshoff Pol, H.E., Hulshoff Pol, H.E., 2009. Efficiency of functional brain networks and intellectual performance. The Journal of Neuroscience. 29, 7619-7624.

Wang, J., Wang, L., Zang, Y., Yang, H., Tang, H., Gong, Q., Chen, Z., Zhu, C., He, Y., 2009. Parcellation-dependent small-world brain functional networks: a resting-state fMRI study. Human Brain Mapping. 30, 1511-1523.

Wang, J., Zuo, X., Dai, Z., Xia, M., Zhao, Z., Zhao, X., Jia, J., Han, Y., He, Y., 2013. Disrupted functional brain connectome in individuals at risk for Alzheimer's disease. Biological Psychiatry. 73, 472-481.

Wang, J., Wang, X., Xia, M., Liao, X., Evans, A., He, Y., 2015. GRETNA: a graph theoretical network analysis toolbox for imaging connectomics. Frontier in Human Neuroscience. 9. http://dx.doi.org/10.3389/fnhum.2015.00386.

Wang, T., Shi, F., Jin, Y., Yap, P.T., Wee, C.Y., Zhang, J., Yang, C., Li, X., Xiao, S., Shen, D., 2016. Multilevel Deficiency of White Matter Connectivity Networks in Alzheimer's Disease: A Diffusion MRI Study with DTI and HARDI Models. Neural Plasticity. 2016, http://dx.doi.org/10.1155/2016/2947136.

Watts, D.J., Strogatz, S.H., 1998. Collective dynamics of small-world networks. Nature. 393, 440-442.

Xia, M., Wang, J., He, Y., 2013. BrainNet Viewer: A Network Visualization Tool for Human Brain Connectomics. PLoS ONE. 8, http://dx.doi.org/10.1371/journal.pone.0068910.

Zhu, W., Wen, W., He, Y., Xia, A., Anstey, K.J., Sachdev, P., 2012. Changing topological patterns in normal aging using large-scale structural networks. Neurobiology of Aging. 33, 899-913.

Zlokovic, B.V., 2005. Neurovascular mechanisms of Alzheimer's neurodegeneration. Trends in Neurosciences. 28, 202-208.

Zuendorf, G., Kerrouche N Fau - Herholz, K., Herholz K Fau - Baron, J.-C., Baron, J.C., 2003. Efficient principal component analysis for multivariate 3D voxel-based mapping of brain functional imaging data sets as applied to FDG-PET and normal aging. Human Brain Mapping. 18, 13-21. 
Figure 1. The correlation coefficient matrixes and binary matrixes of the two groups. (A) The partial correlation coefficient matrix (indicated by the color bar, ranging from -1.0 to 1.0) of $\mathrm{HC}$ group. (B) The partial correlation coefficient matrix of AD group. (C) The binary matrix of HC group thresholded at a fixed density $15 \%$. (D) The binary matrix of AD group thresholded at a fixed density $15 \%$.

Figure 2. Comparison between networks with and without regressing out the factors of sex, age and weight. The first row represents the results of clustering coefficient, path length and sigma of HC group; The second row represents the results of clustering coefficient, path length and sigma of $\mathrm{AD}$ group; The third row represents the difference between two groups in clustering coefficient, path length and sigma; blue line: without regressing; red line: with regressing.

Figure 3. Network parameters of gamma, lambda, and sigma in the AD group and HC group. (A) gamma parameters; (B) lambda parameters; (C) sigma parameters. The $\mathrm{x}$-coordinate represents the sparsity threshold that ranges from $6 \%$ to $40 \%$ and increases in $1 \%$ steps for a total of 35 thresholds. The y-coordinate represents the network parameter values. The red curve refers to HC, and the blue curve refers to AD. Note: for gamma, lambda, and sigma, the differences between the $\mathrm{HC}$ and $\mathrm{AD}$ groups were significant over the entire threshold range of $6 \%-40 \%$ ( $<$ $0.05)$.

Figure 4. Network parameters of $\mathrm{C}$, $\mathrm{L}$, globalE, and localE in the $\mathrm{AD}$ group and HC group. (A) C, clustering coefficient; (B) L, characteristic path length; (C) globalE, global efficiency; (D) localE, local efficiency. Note: * represents significant differences between the HC and AD groups at the sparsity threshold ( $p<0.05)$.

Figure 5. The size of the largest connected component of two networks. When the threshold increases, the largest component sizes of both groups tend to increase. The arrow indicates that the lowest sparsity threshold (18\% in HC, $13 \%$ in $\mathrm{AD}$ ) in which both of the networks included all connected nodes.

Figure 6. 21 hub nodes in the HC group (left, blue circle) and 18 hub nodes in the AD group (right, red circle). The node's size was set according to its value of normalized centrality. The hub nodes were visualized using the BrainNet Viewer package (Xia et al., 2013), showing the brain model in the axial view (dorsal side).

Figure 7. Altered hubs in the AD group. The red circles indicate regions where the AD group showed increased betweenness centrality as compared with HC. The blue circles indicate regions where the AD network showed decreased betweenness centraility as compared with HC. L refers to the left hemispheres, and R refers to the right hemispheres.

Figure 8. The bi values and the $p$ values of the three altered hubs. The blue bar chart represents HC group; the red bar chart represents AD group. From left to right, SFGdor.L, CUN.L and CAU.R. 
Figure 9. Seed ROI-based correlation associated with the CUN.L region. (A) correlation coefficients map associated with the CUN.L seed in the HC group; (B) correlation coefficients map associated with the CUN.L seed in the AD group; (C) Z-statistics map showing the brain regions that had strengthened connection with the CUN.L seed in AD as compared to HC ( $\mathrm{p}<0.05$, FDR corrected); and (D) The location of CUN.L seed in AAL template. The results were performed with the REST Slice Viewer (Song et al., 2011).

Figure 10. Seed ROI-based correlation associated with the CAU.R region. (A) correlation coefficients map associated with the CAU.R seed in the HC group; (B) correlation coefficients map associated with the CAU.R seed in the AD group; (C) Z-statistics map showing the brain regions that had strengthened connection with the CAU.R seed in AD as compared to HC ( $\mathrm{p}<0.05$, FDR corrected); and (D) The location of CAU.R seed in AAL template.

Figure 11. ROC curve of the altered hubs and whole-brain for AD classification. The $\mathrm{x}$-coordinate represents the false positive rate, and the y-coordinate represents the true positive rate. 


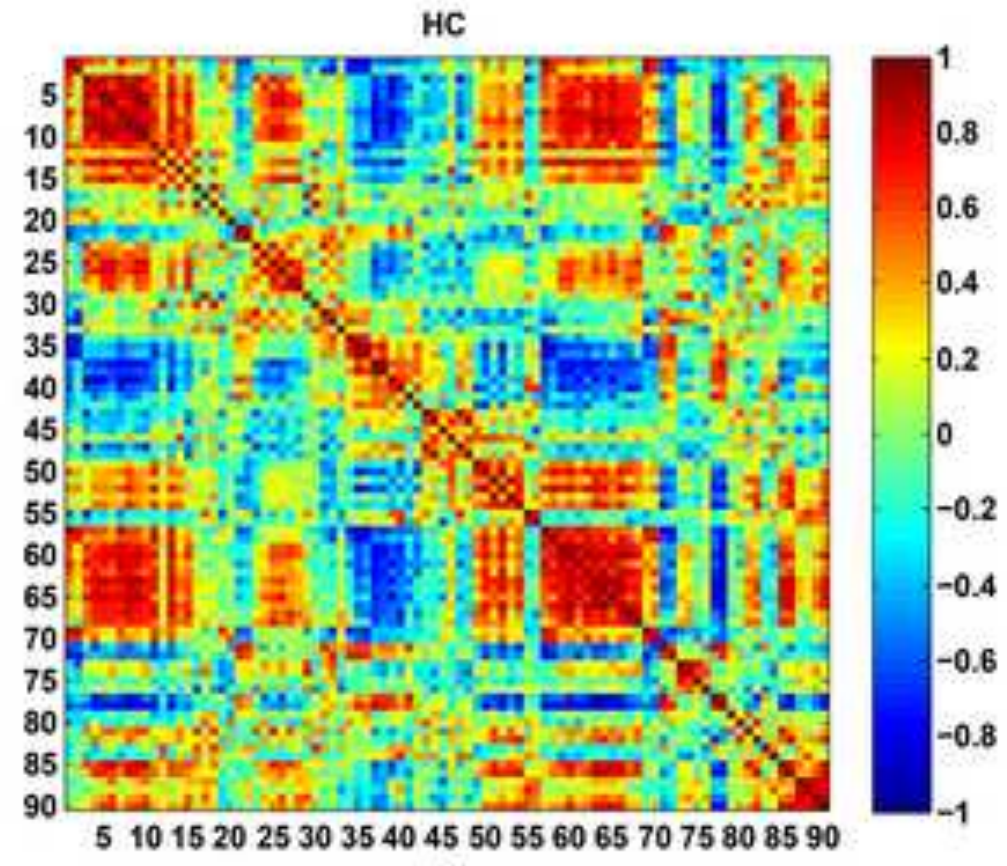

A

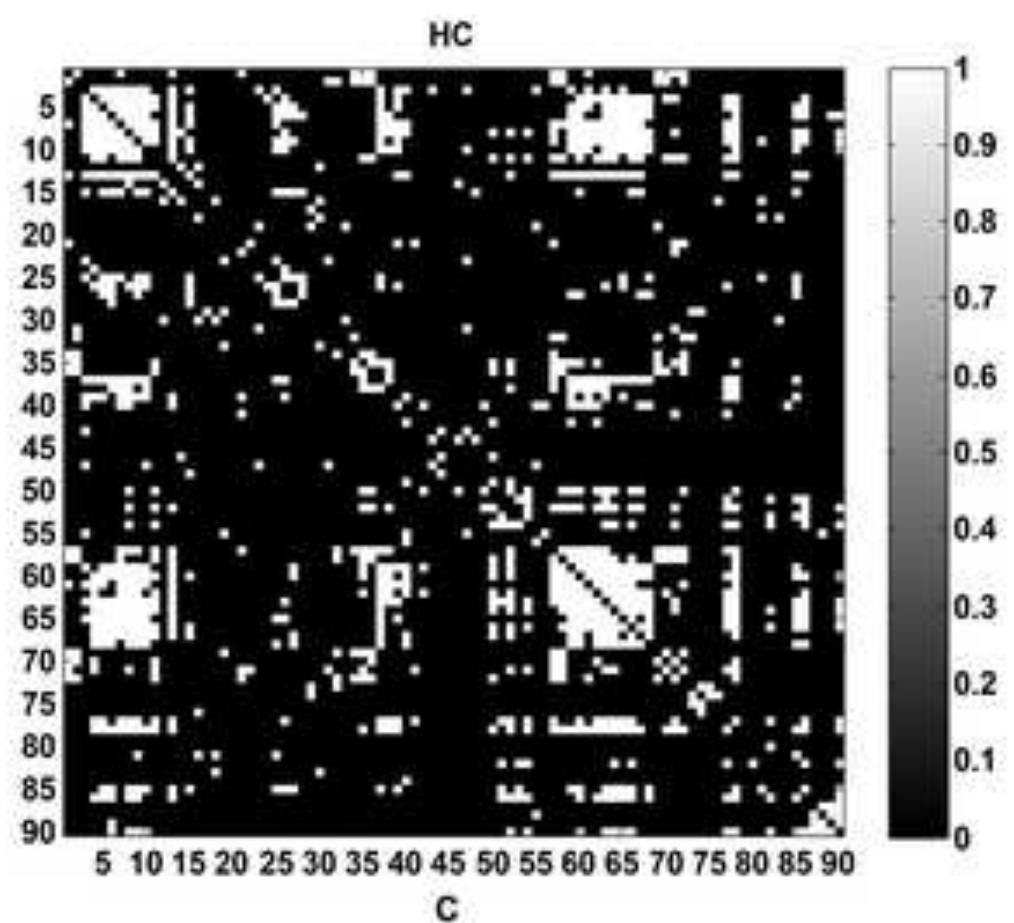

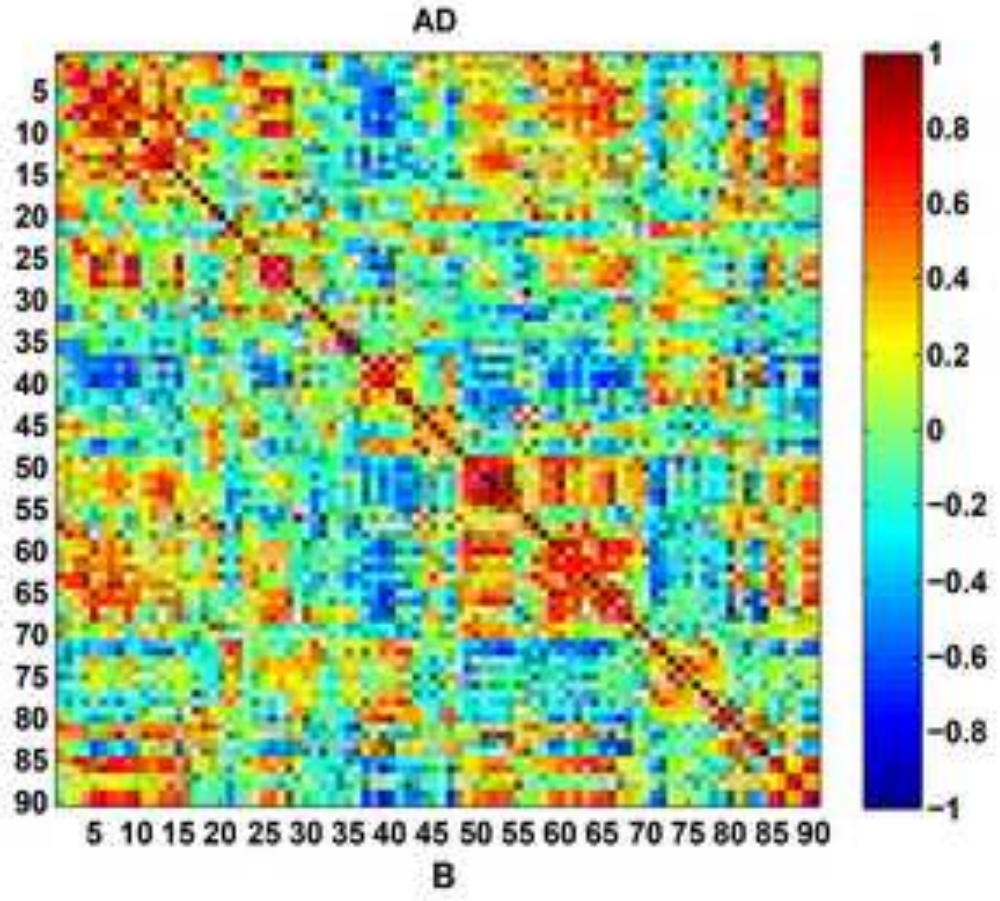

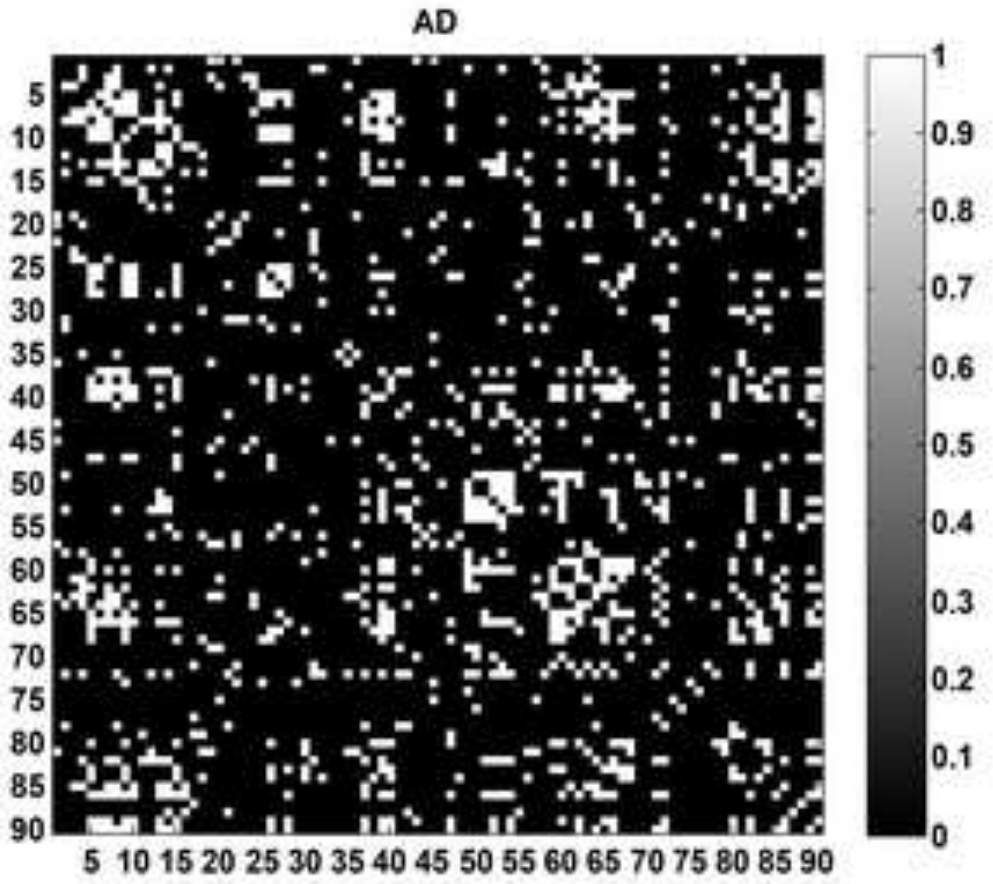

D 

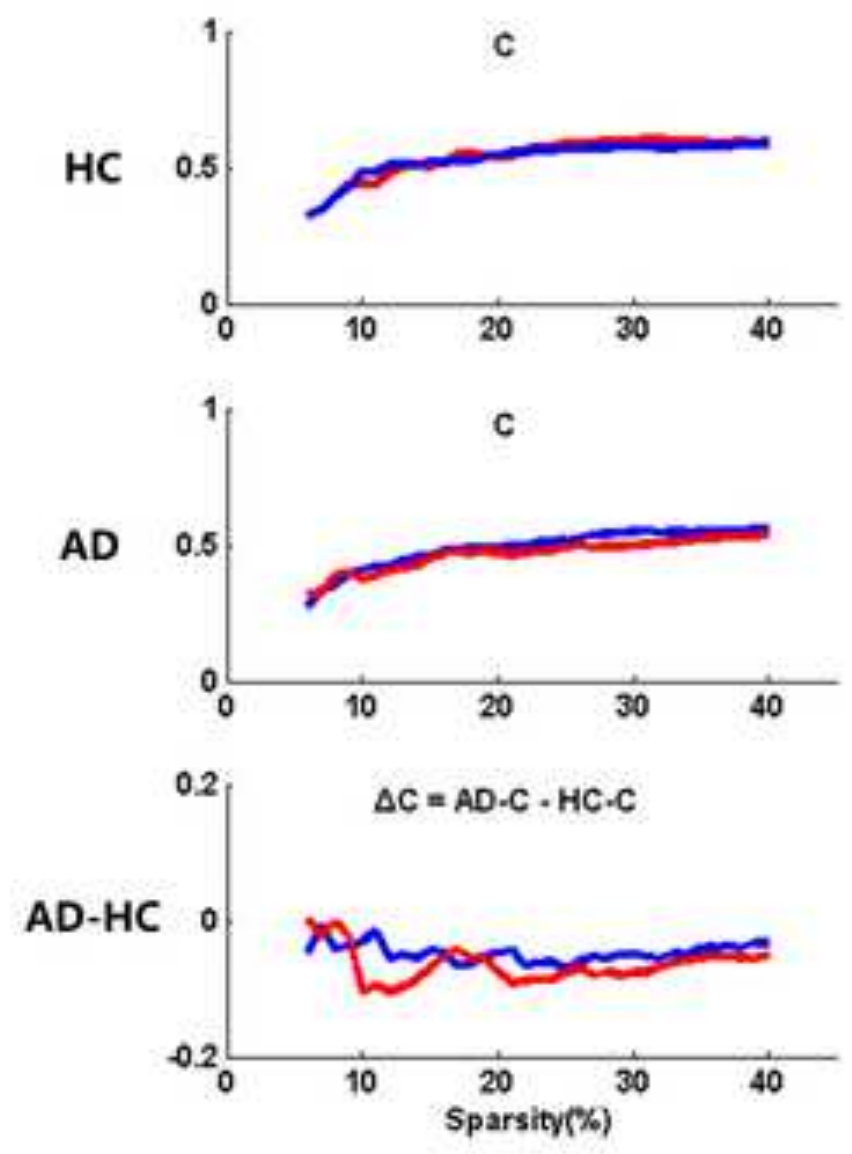
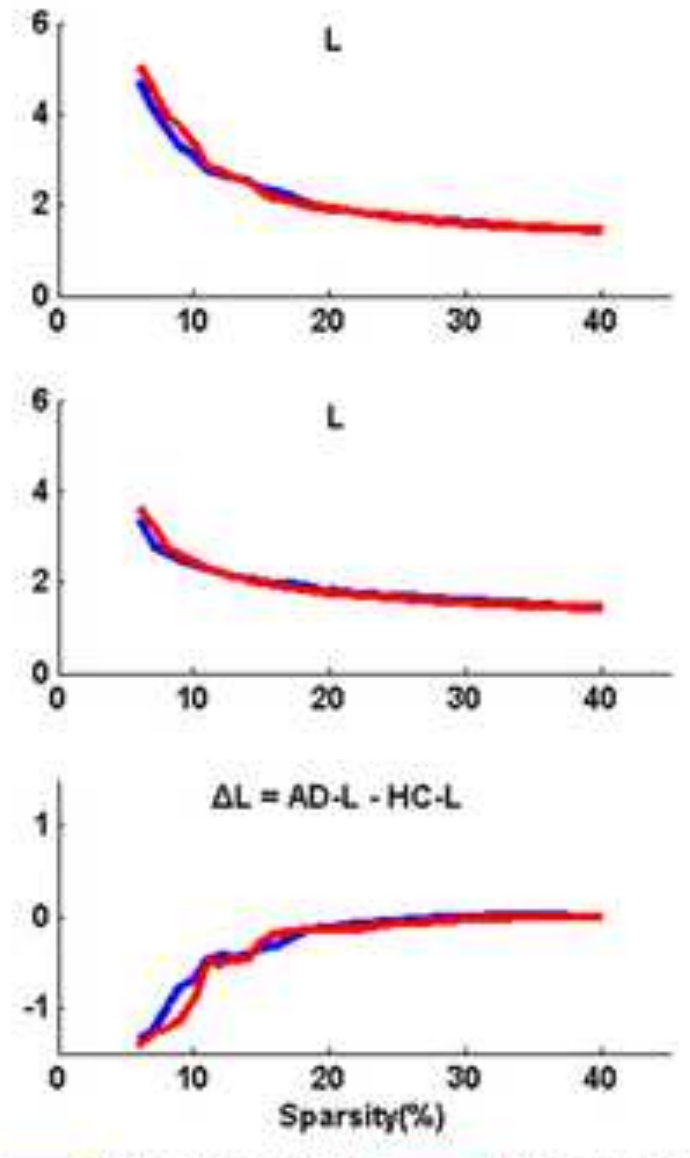

- without regressing - with regressing
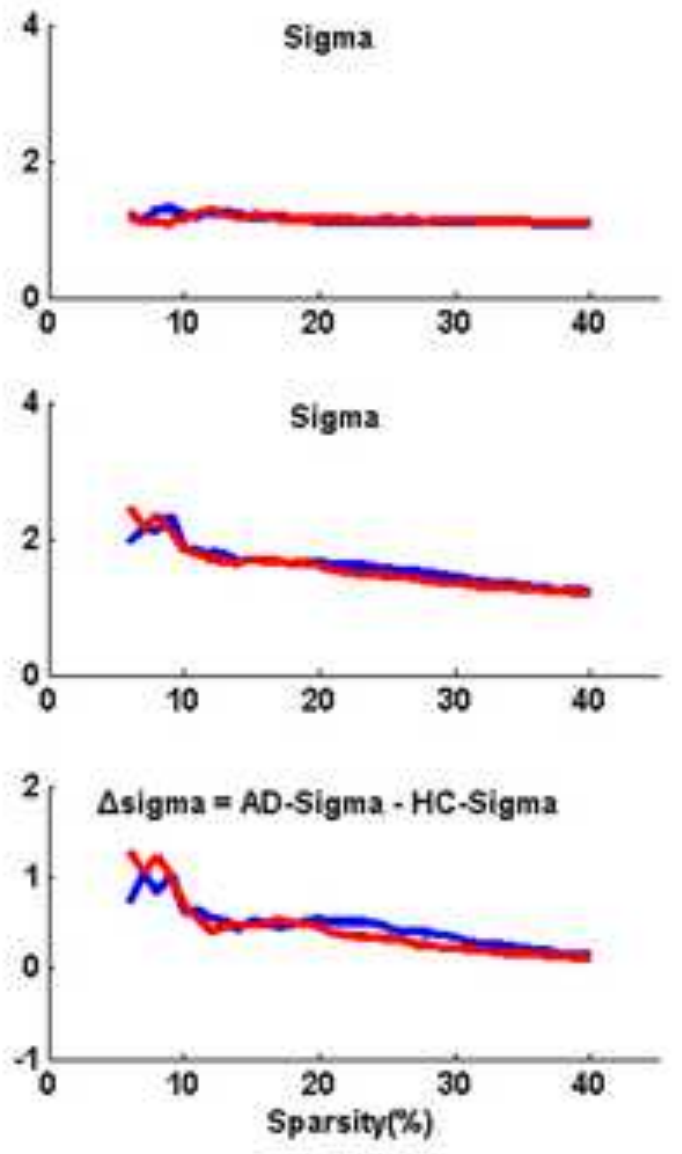


\section{Figure 3}
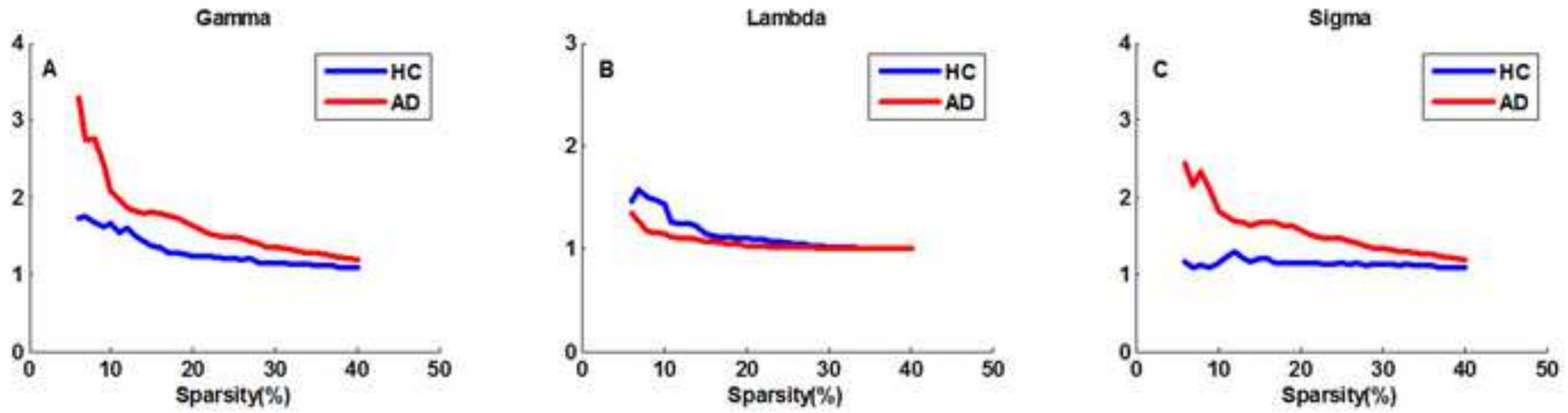

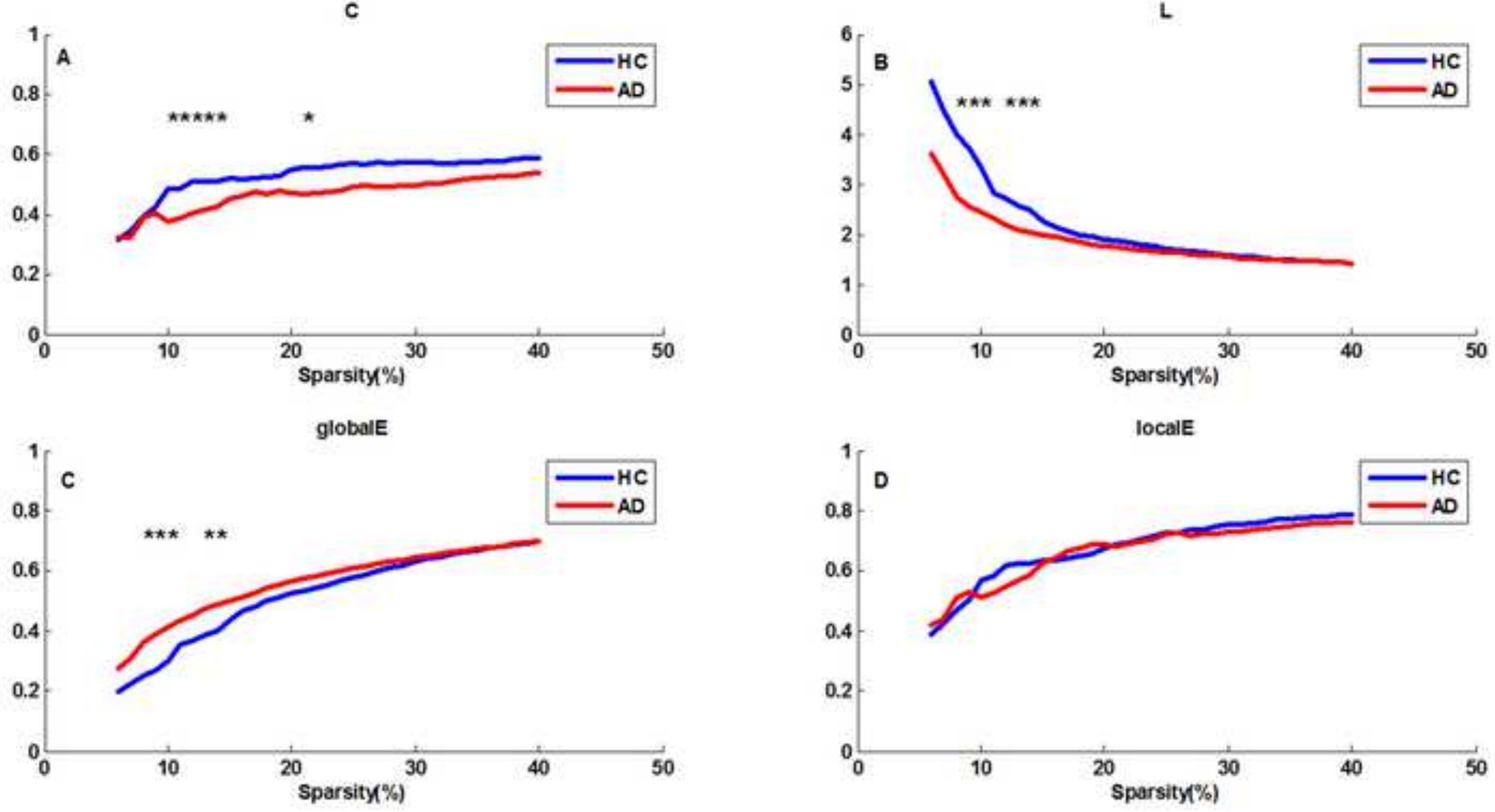


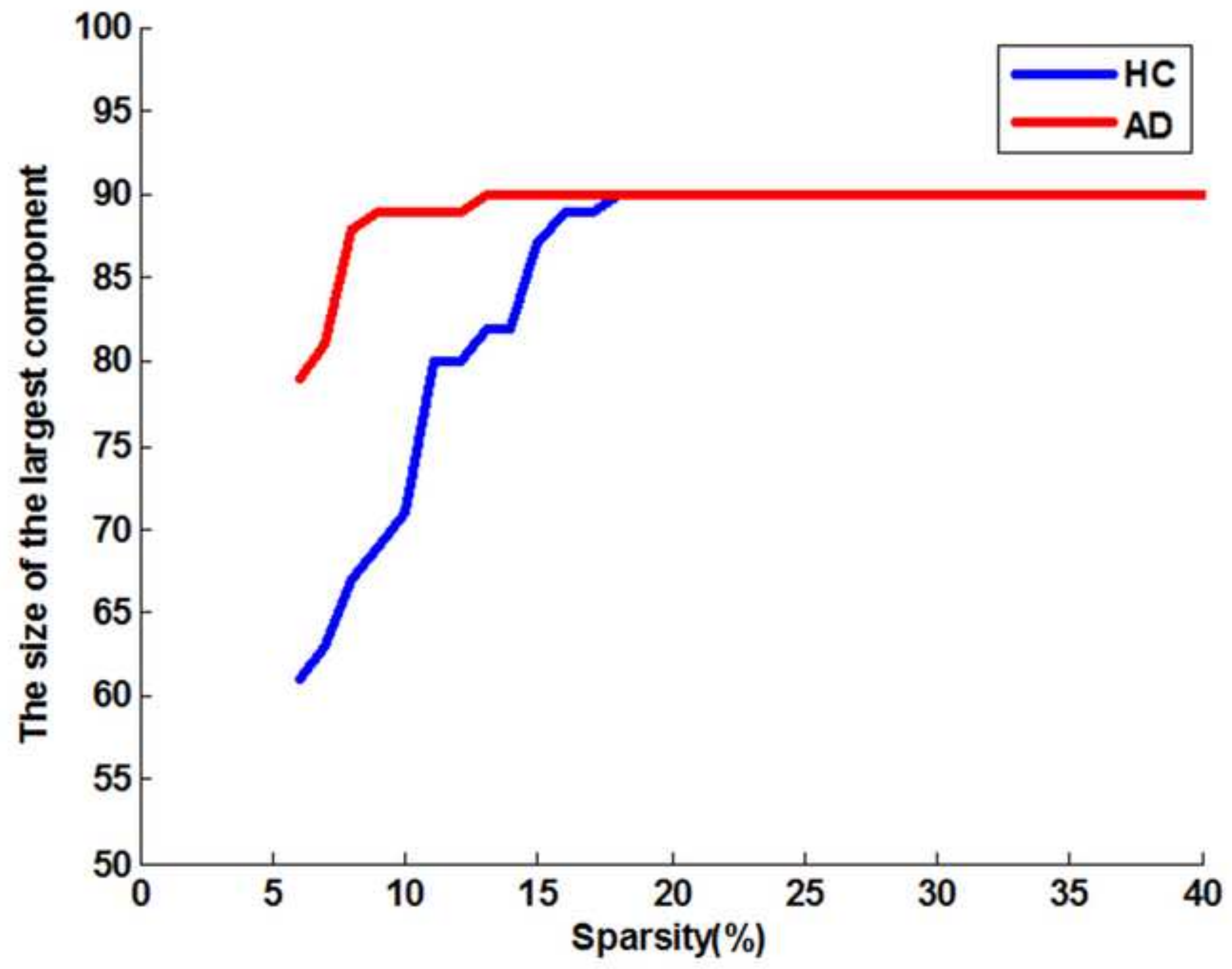




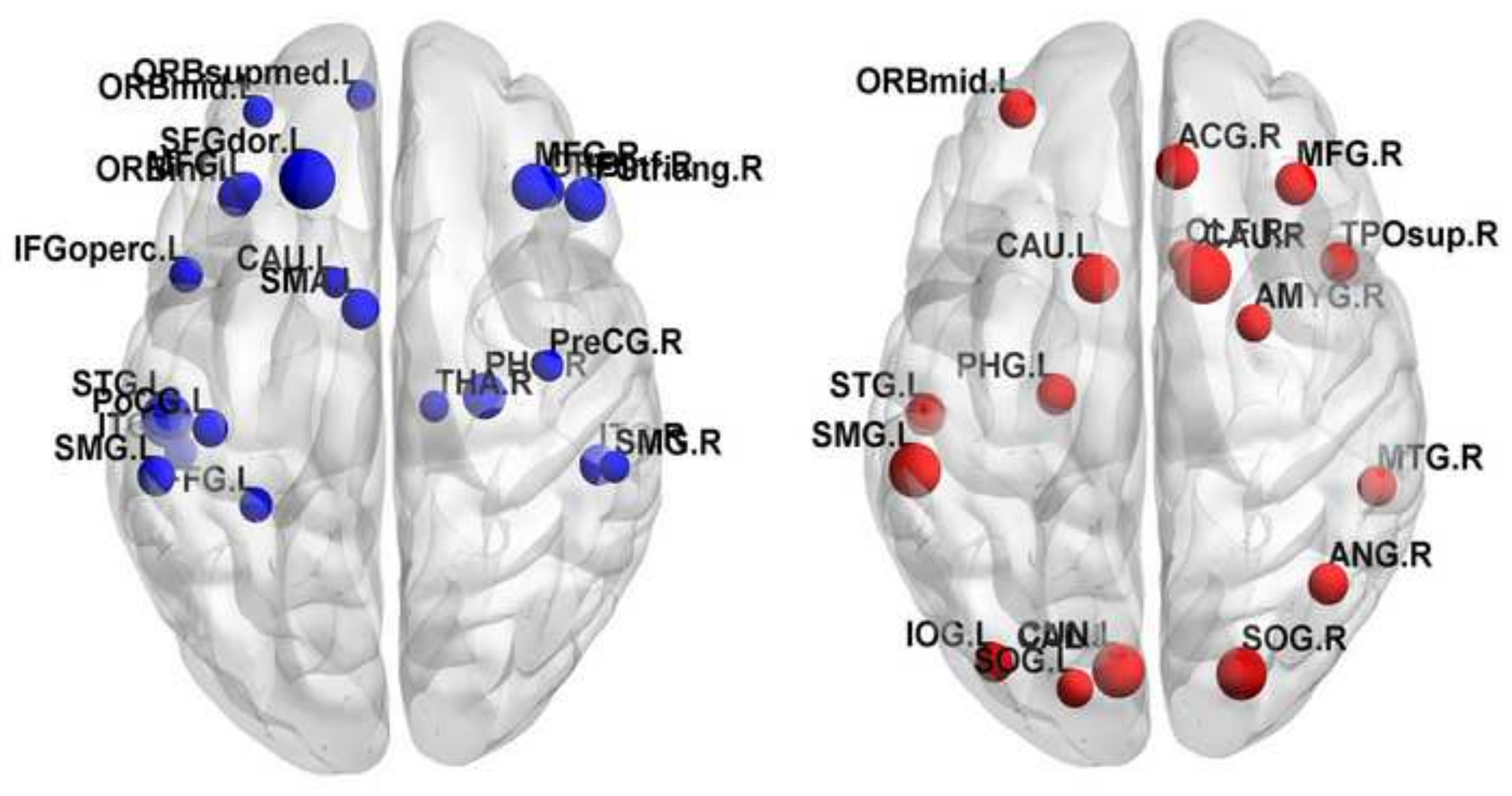


L

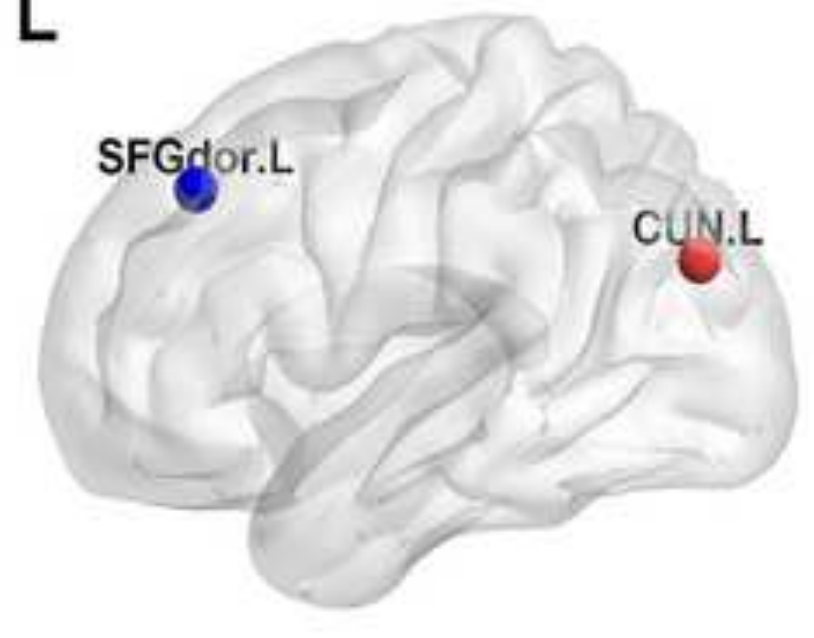

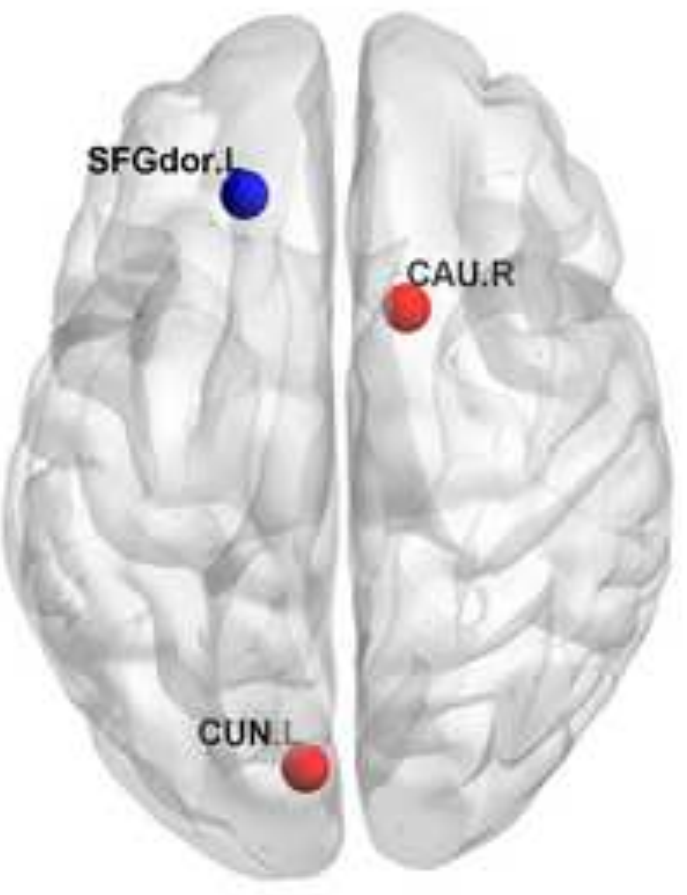

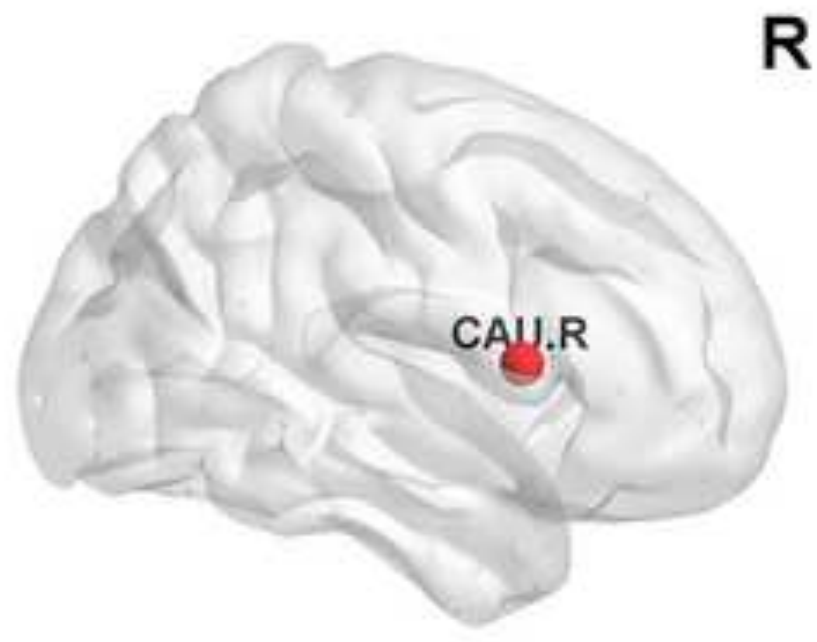

R 


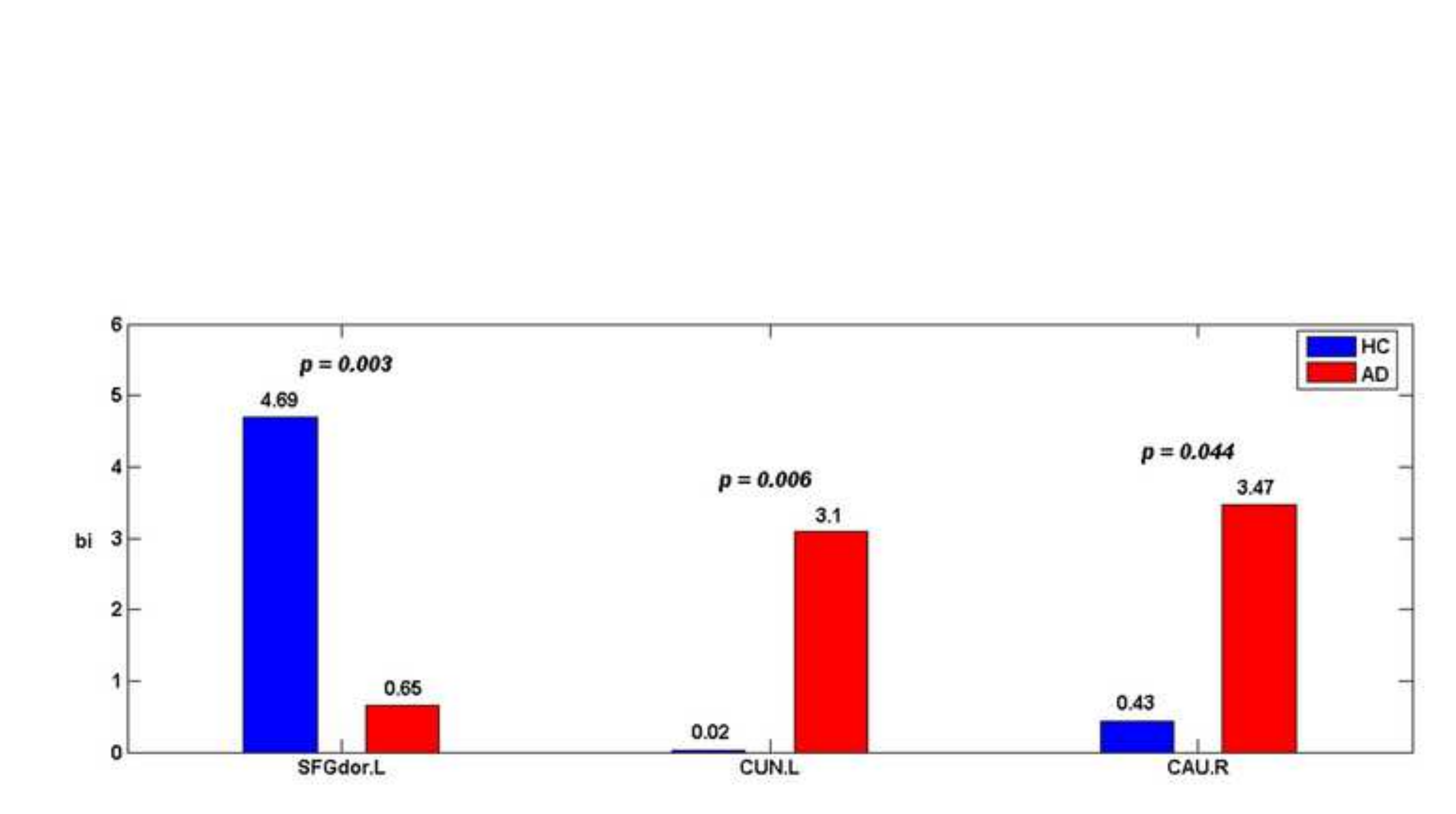

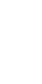

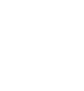
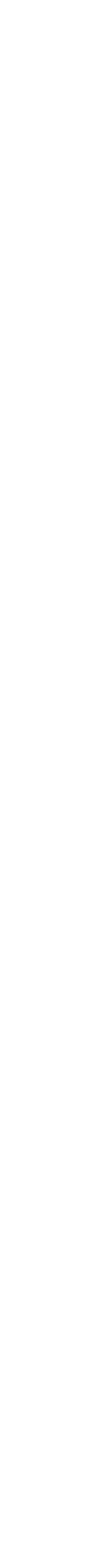

.

(

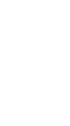

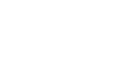



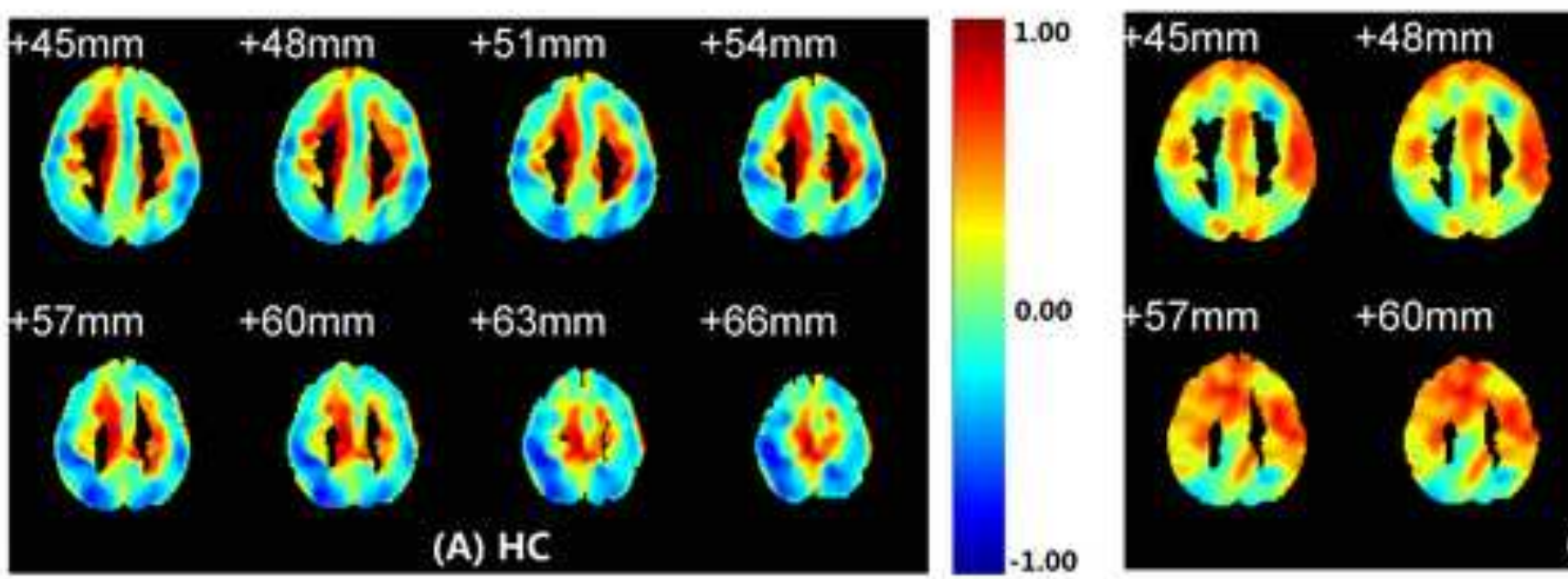

$+51 \mathrm{~mm}$
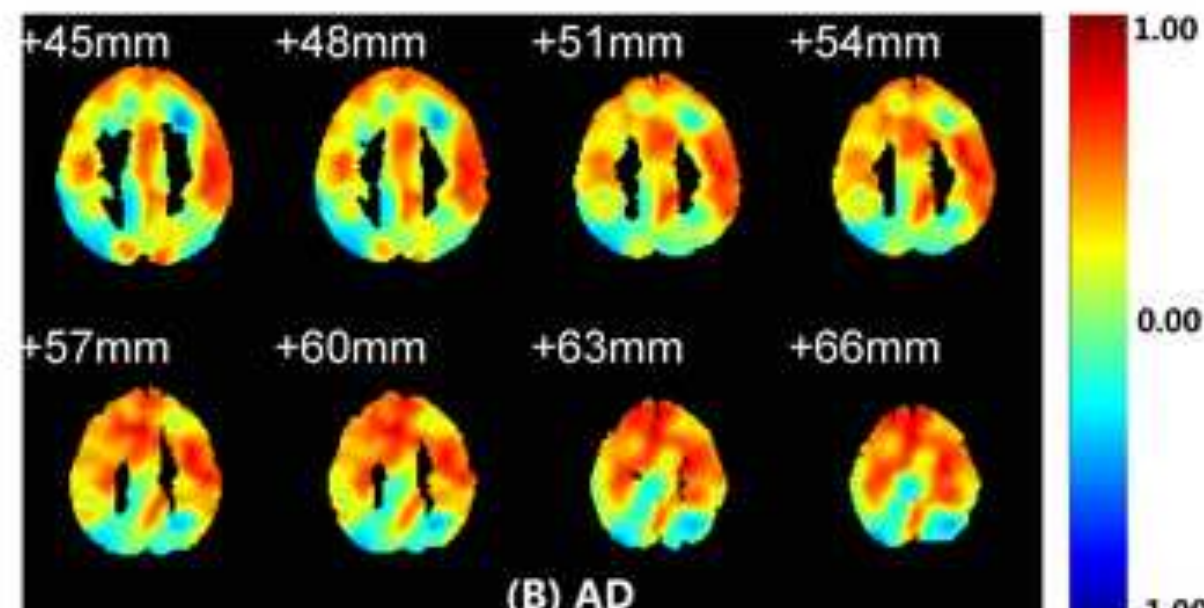

(B) AD
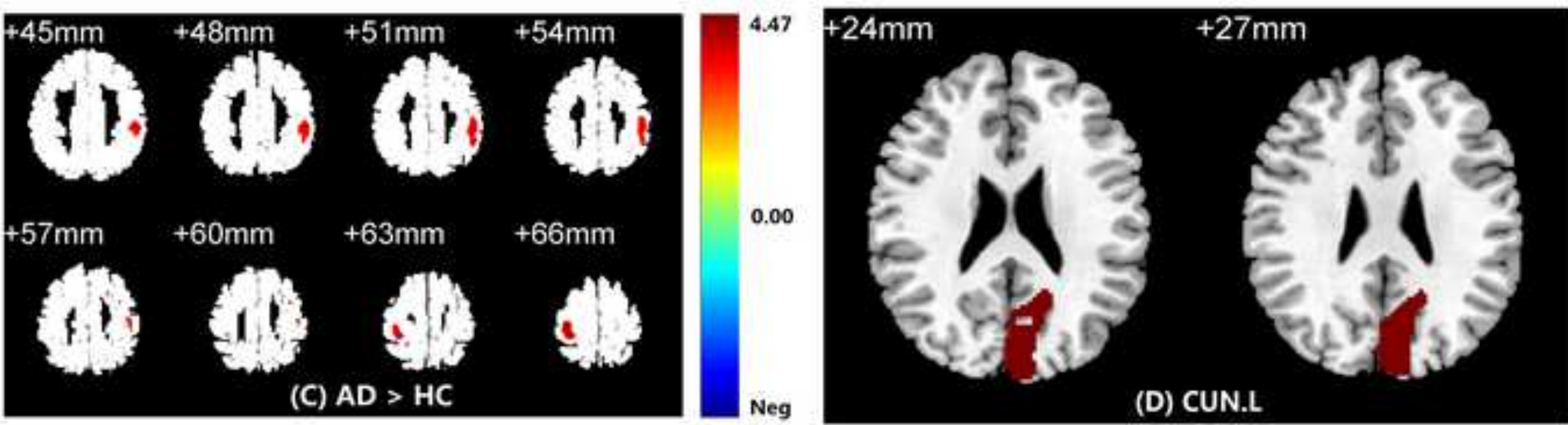

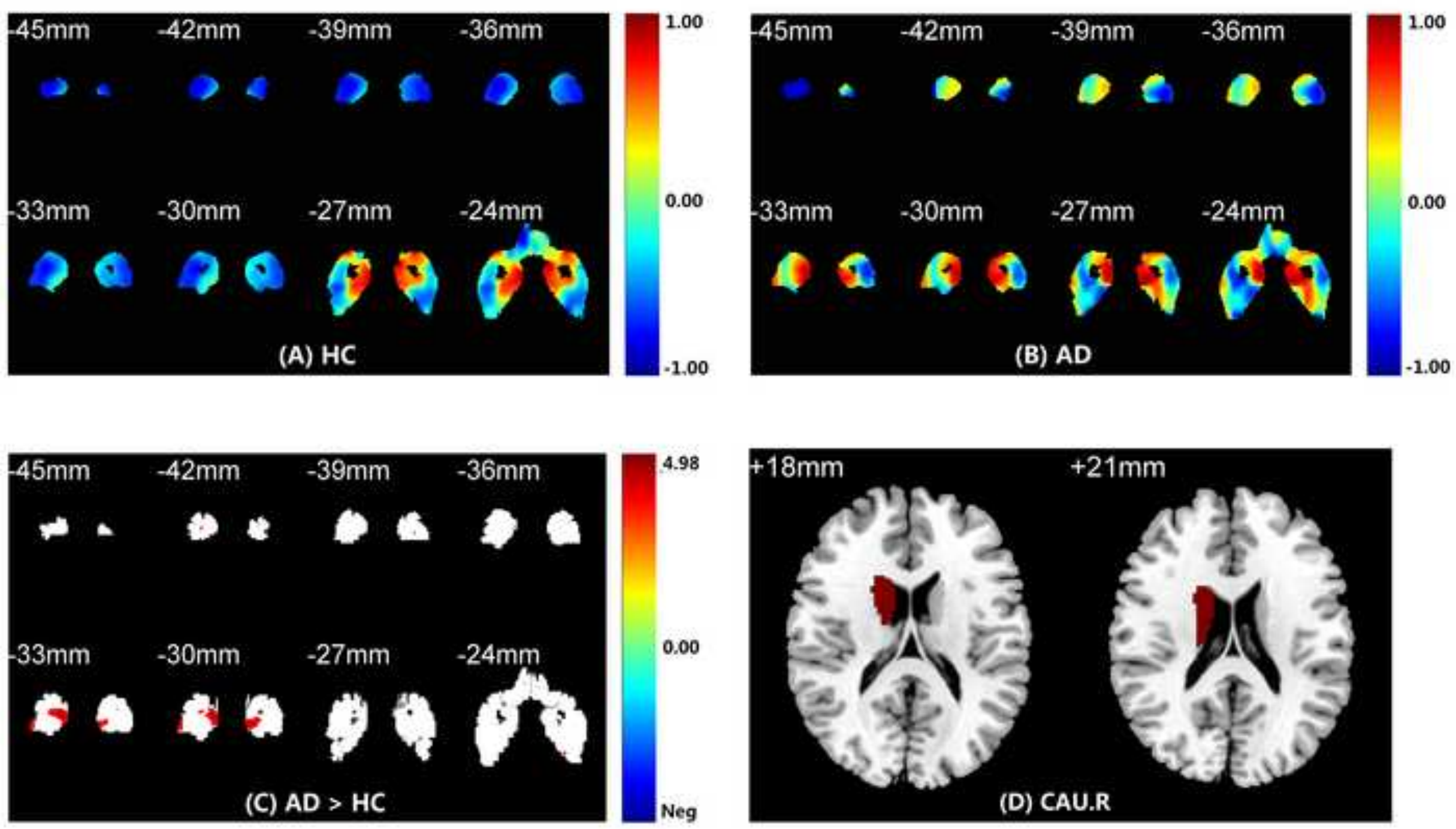
ROC curves

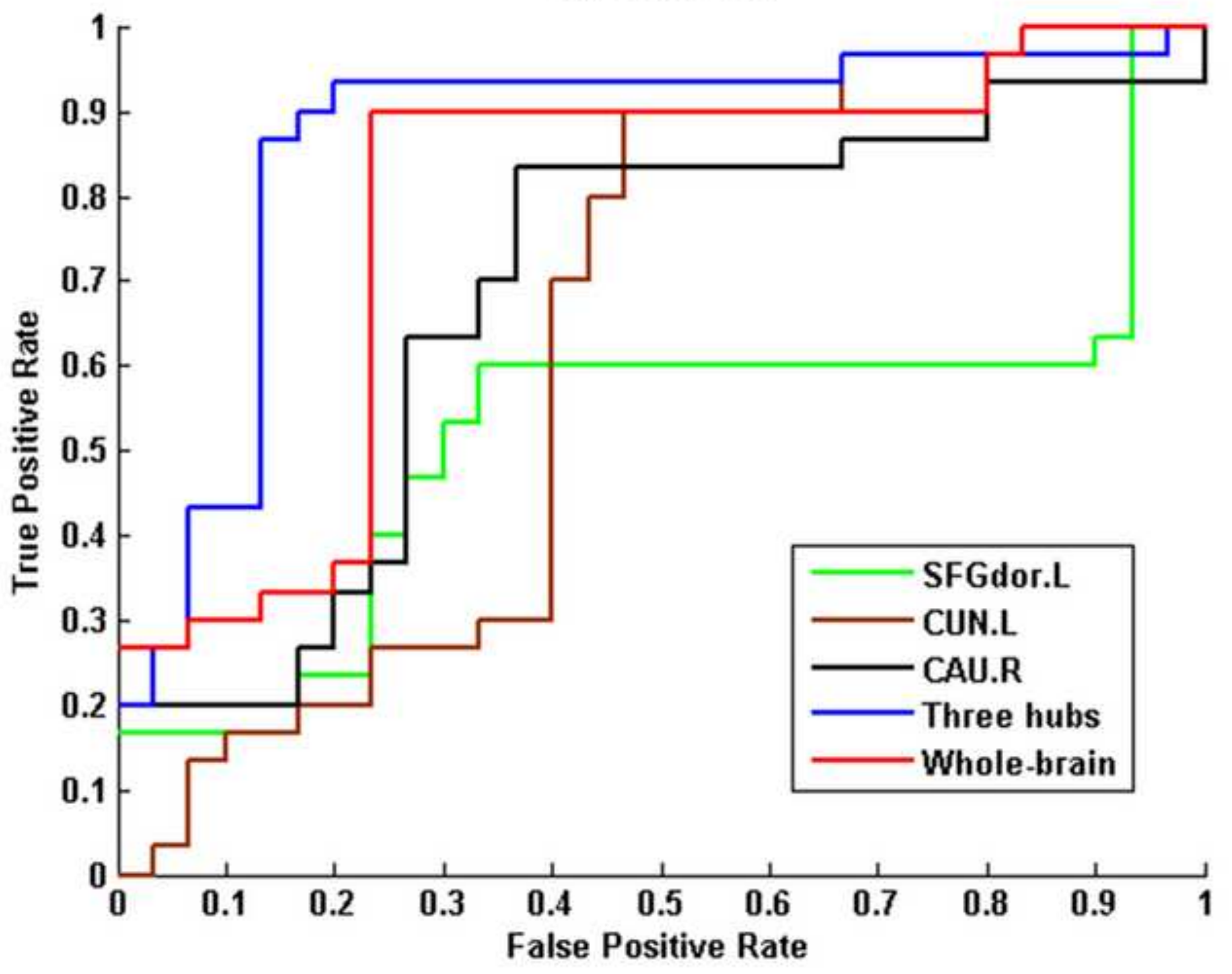




\section{Tables}

Table 1. The statistical information of the 34 participants

\begin{tabular}{c|cccc}
\hline \multicolumn{1}{c}{ Data } & Info & HC group & AD group & P value \\
\hline \multirow{4}{*}{ Dataset 1 } & Gender (M/F) & $5 \mathrm{~F} \mathrm{11M}$ & $10 \mathrm{~F} 8 \mathrm{M}$ & $0.15^{\mathrm{a}}$ \\
16 HC, 18 AD & Age (years) & $72.0 \pm 5.5$ & $69.8 \pm 9.8$ & $0.41^{\mathrm{b}}$ \\
& Weight (kg) & $65.5 \pm 11.1$ & $58.3 \pm 10.3$ & $0.06^{\mathrm{b}}$ \\
& MMSE & $29.3 \pm 1.2$ & $18.8 \pm 3.8$ & $<0.001^{\mathrm{b}}$ \\
& CDR(n) & $0(6)$ & $0.5(14), 1(3), 2(1)$ & $<0.001^{\mathrm{b}}$ \\
\hline \multirow{5}{*}{ Dataset 2 } & Gender (M/F) & $20 \mathrm{~F} \mathrm{10M}$ & $16 \mathrm{~F} \mathrm{14M}$ & $0.29^{\mathrm{a}}$ \\
& Age (years) & $77.0 \pm 6.9$ & $75.4 \pm 8.8$ & $0.43^{\mathrm{b}}$ \\
& Weight (kg) & $73.7 \pm 12.1$ & $73.6 \pm 13.8$ & $0.98^{\mathrm{b}}$ \\
& MMSE & $30 \pm 0$ & $19.7 \pm 6.6$ & $<0.001^{\mathrm{b}}$ \\
& CDR(n) & $0(30)$ & $0.5(17), 1(9), 2(4)$ & $<0.001^{\mathrm{b}}$ \\
\hline
\end{tabular}

Note: Data is presented as the mean \pm standard deviation.

$\mathrm{AD}$, Alzheimer's disease; HC, healthy control; F, females; $\mathrm{M}$, males; $\mathrm{n}$ refers to the number of samples. In Dataset 1, only six subjects provided the MMSE and CDR. ${ }^{\mathrm{a}} \mathrm{P}$-value was obtained by the Pearson chi-square test. ${ }^{\mathrm{b}} \mathrm{P}$-value was obtained by the two-sample two-tailed t-test. 
Table 2. The summary of network parameters

\begin{tabular}{|c|c|c|c|}
\hline parameters & abbreviate & equations & meaning \\
\hline $\begin{array}{l}\text { clustering } \\
\text { coefficient }\end{array}$ & $\mathrm{C}$ & $\begin{array}{c}\mathrm{C}=\frac{1}{N} \sum_{i=1}^{N} \frac{2 E(i)}{k_{i}\left(k_{i}-1\right)} \\
\mathrm{N} \text { : number of nodes } \\
\mathrm{K}_{\mathrm{i} \cdot \text { number of nodes connected with node } \mathrm{i}}^{\mathrm{E}(\mathrm{i}): \text { actual connections edge among } \mathrm{k}_{\mathrm{i}}}\end{array}$ & $\begin{array}{l}\text { measures the degree to } \\
\text { which nodes in a graph } \\
\text { tend to cluster together }\end{array}$ \\
\hline $\begin{array}{c}\text { characteristic } \\
\text { path length }\end{array}$ & $\mathrm{L}$ & $\begin{array}{c}\mathrm{L}=\frac{1}{\frac{1}{N(N-1)} \sum_{i, j \in V, i \neq j} \frac{1}{d_{i j}}} \\
\mathrm{~N} \text { : number of nodes in network } \mathrm{V} \\
\mathrm{d}_{\mathrm{ij}}: \text { the shortest path between node } \mathrm{i} \text { and } \mathrm{j}\end{array}$ & $\begin{array}{l}\text { a measure of the } \\
\text { efficiency of the } \\
\text { information or mass } \\
\text { transport on a network }\end{array}$ \\
\hline gamma & gamma & $\begin{array}{c}\text { gamma }=\frac{C}{C_{\text {rand }}} \\
\mathrm{C}_{\text {rand }}: \text { clustering coefficient of corresponding } \\
\text { random network }\end{array}$ & $\begin{array}{l}\text { a network could be } \\
\text { defined as small-world }\end{array}$ \\
\hline $\begin{array}{l}\text { small-world } \\
\text { coefficient }\end{array}$ & lambda & $\begin{array}{c}\text { lambda }=\frac{L}{L_{\text {rand }}} \\
\mathrm{L}_{\text {rand }}: \text { path length of corresponding } \\
\text { random network } \\
\text { sigma }=\frac{\text { gamma }}{\text { lambda }}\end{array}$ & $\begin{array}{c}\text { following criteria: } \\
\text { gamma }>1 \\
\text { lambda } \approx 1 \\
\text { sigma }>1\end{array}$ \\
\hline $\begin{array}{c}\text { global } \\
\text { efficiency }\end{array}$ & globalE & globalE $=\frac{1}{N(N-1)} \sum_{i, j \in V, i \neq j} \frac{1}{d_{i j}}$ & \\
\hline $\begin{array}{c}\text { local } \\
\text { efficiency }\end{array}$ & localE & $\begin{array}{c}\text { localE }= \\
\frac{1}{N} \sum_{k \in V}\left(\frac{1}{N_{v k}\left(N_{v k}-1\right)} \sum_{i, j \in V, i \neq j} \frac{1}{d_{i j}}\right) \\
\mathrm{N}_{\mathrm{vk}}: \text { subgraph of node } \mathrm{k}\end{array}$ & $\begin{array}{l}\text { efficiently the network } \\
\text { exchanges information }\end{array}$ \\
\hline $\begin{array}{c}\text { betweenness } \\
\text { centrality }\end{array}$ & $\mathrm{BC}$ & $\begin{array}{c}B C_{k}=\sum_{i, j \in V, i \neq j} \frac{n_{i j}(k)}{n_{i j}} \\
\mathrm{n}_{\mathrm{ij}}(\mathrm{k}) \text { :shortest path pass between nodes } \mathrm{i} \text { and } \mathrm{j} \\
\text { through node } \mathrm{k}\end{array}$ & $\begin{array}{l}\text { an indicator of a } \\
\text { node's centrality in } \\
\text { a network }\end{array}$ \\
\hline
\end{tabular}


Table 3. The hub regions of $\mathrm{HC}$ group

\begin{tabular}{lll}
\hline hubs & class & bi \\
\hline PreCG.R & Primary & 1.57 \\
SFGdor.L & Association & 4.69 \\
MFG.L & Association & 1.65 \\
MFG.R & Association & 3.25 \\
ORBmid.L & Paralimbic & 1.67 \\
IFGoperc.L & Association & 2.04 \\
IFGtriang.R & Association & 3.04 \\
ORBinf.L & Paralimbic & 2.49 \\
ORBinf.R & Paralimbic & 1.89 \\
SMA.L & Association & 2.57 \\
ORBsupmed.L & Paralimbic & 1.53 \\
PHG.R & Paralimbic & 3.20 \\
FFG.L & Association & 1.96 \\
PoCG.L & Primary & 2.26 \\
SMG.L & Association & 2.54 \\
SMG.R & Association & 1.65 \\
CAU.L & Subcortical & 1.54 \\
THA.R & Subcortical & 1.58 \\
STG.L & Association & 4.20 \\
ITG.L & Association & 1.99 \\
ITG.R & Association & 2.61 \\
\hline
\end{tabular}


Table 4. The hub regions of AD group

\begin{tabular}{lll}
\hline hubs & class & bi \\
\hline MFG.R & Association & 2.15 \\
ORBmid.L & Paralimbic & 1.80 \\
OLF.R & Limbic & 1.53 \\
ACG.R & Paralimbic & 2.31 \\
PHG.L & Paralimbic & 2.01 \\
AMYG.R & Subcortical & 1.70 \\
CAL.L & Primary & 1.73 \\
CUN.L & Association & 3.10 \\
SOG.L & Association & 1.76 \\
SOG.R & Association & 2.93 \\
IOG.L & Association & 1.85 \\
SMG.L & Association & 3.07 \\
ANG.R & Association & 2.05 \\
CAU.L & Subcortical & 2.64 \\
CAU.R & Subcortical & 3.47 \\
STG.L & Association & 2.09 \\
TPOsup.R & Paralimbic & 1.93 \\
MTG.R & Association & 1.97 \\
\hline
\end{tabular}

Table 5. The classification performance of the three altered hubs and the whole-brain

\begin{tabular}{|c|c|c|c|c|c|}
\hline Biomarkers & Validation & $\operatorname{Sen}(\%)$ & Spc $(\%)$ & $\operatorname{Acc}(\%)$ & AUC \\
\hline SFGdor.L & \multirow{2}{*}{ Features: PCs } & 60.0 & 66.7 & 63.3 & 0.52 \\
\hline CUN.L & & 56.7 & 60.0 & 58.3 & 0.63 \\
\hline CAU.R & \multirow{3}{*}{$\begin{array}{l}\text { Kernel: sigmoid } \\
\text { Method: Leave-one-out }\end{array}$} & 83.3 & 63.3 & 73.3 & 0.68 \\
\hline $\begin{array}{c}\text { Three } \\
\text { altered hubs }\end{array}$ & & 90.0 & 80.0 & 85.0 & 0.86 \\
\hline Whole-brain & & 90.0 & 76.7 & 83.3 & 0.78 \\
\hline
\end{tabular}

